\title{
Atlas of monochromatic images of planetary nebulae ${ }^{\star}$ (Research Note)
}

\author{
W. A. Weidmann ${ }^{1,2, \star \star}$, E. O. Schmidt ${ }^{3}$, R. R. Vena Valdarenas ${ }^{3}$, J. A. Ahumada ${ }^{1}$, M. G. Volpe ${ }^{1}$, and A. Mudrik ${ }^{4}$ \\ 1 Observatorio Astronómico, Universidad Nacional de Córdoba, Laprida 854, 5000 Córdoba, Argentina \\ e-mail: walter@oac.unc.edu.ar \\ 2 Consejo Nacional de Investigaciones Científicas y Técnicas (CONICET), Av. Int. Güiraldes 2620, C14282AA, \\ Ciudad Autónoma de Buenos Aires, Argentina \\ 3 Instituto de Astronomía Teórica y Experimental (IATE), Universidad Nacional de Córdoba, CONICET, \\ Observatorio Astronómico de Córdoba, Laprida 854, X5000BGR, Córdoba, Argentina \\ 4 Facultad de Matemática, Astronomía y Física, Universidad Nacional de Córdoba, X5000HUA, Córdoba, Argentina
}

Received 14 August 2015 / Accepted 25 May 2016

\begin{abstract}
We present an atlas of more than one hundred original images of planetary nebulae (PNe). These images were taken in a narrow-band filter centred on the nebular emission of the [N II] during several observing campaigns using two moderate-aperture telescopes, at the Complejo Astronómico El Leoncito (CASLEO), and the Estación Astrofísica de Bosque Alegre (EABA), both in Argentina. The data provided by this atlas represent one of the most extensive image surveys of PNe in [N II]. We compare the new images with those available in the literature, and briefly describe all cases in which our [N II] images reveal new and interesting structures.
\end{abstract}

Key words. planetary nebulae: general - atlases - catalogs - stars: mass-loss

\section{Introduction}

Currently, about 3000 Galactic planetary nebulae (PNe) are known, which is more than twice as many as a decade ago; unfortunately, most of them still remain poorly studied. The PNe are highly representative of the latest stages of stellar evolution for stars of between 0.8 and $8 M_{\odot}$; however, there are still many details of their evolutionary scheme such as the mass-loss processes, which are not yet fully understood. In this context, an extensive and complete atlas of narrow-band images centred on different nebular lines is essential because these images trace the distribution of ionized mass and also provide information about the processes of mass ejection involved in PN formation.

Clearly, it is important for any study about extended astronomical objects, and in particular for a study of PNe, to have access to a sample of narrow-band images that is as complete as possible. However, this type of work is time-consuming, which makes it an ideal project for medium-sized telescopes like those of the Complejo Astronómico El Leoncito (CASLEO) and the Estación Astrofísica de Bosque Alegre (EABA).

Despite the importance of these narrow-band surveys, there are not many works of this kind. In addition, only a few studies contain observations in [N II] and in general cover only a small number of objects. The most relevant previous catalogues containing the [N II] narrow band that have been published are those by Balick (1987, 50 objects),

\footnotetext{
$\star$ The reduced images as FITS files are only available at the CDS via anonymous ftp to cdsarc.u-strasbg. $f r(130.79 .128 .5)$ or via http://cdsarc.u-strasbg.fr/viz-bin/qcat?J/A+A/592/A103 $\star \star$ Visiting Astronomer, Complejo Astronómico El Leoncito operated under agreement between the Consejo Nacional de Investigaciones Científicas y Técnicas de la República Argentina and the National Universities of La Plata, Córdoba and San Juan.
}

Manchado et al. (1996, 243 objects), Tweedy \& Kwitter (1996, 19 objects), Hua et al. (1998, 22 objects), Górny et al. (1999, 101 objects) and Hajian et al. (2007, 18 objects).

Here we present an atlas of images comprising $108 \mathrm{PNe}$ in the $[\mathrm{N} \mathrm{II}]$ narrow band. The goal is to increase the amount of observational data of these amazing and highly complex objects. We hope that this work (together with the previous ones) will become a useful database for future research.

\section{Sample, observations, and data reduction}

We have observed a sample of 108 true PNe selected from the catalogues of Acker et al. (1992) and Parker et al. (2005, 2006). The selected nebulae did not have previous observations in [N II], except for a small group that was chosen to compare the quality of our data with that of previous works. Our selection criterion requires that the angular size of the objects in the mentioned catalogues be between 16 and 200 arcsec. In this way, it should be possible to infer its morphology and, in some cases, to detect low-ionization regions (LIS).

The observational data were obtained with the $1.54 \mathrm{~m}$ telescope at EABA (configured in the newtonian focus) and the $2.15 \mathrm{~m}$ telescope at CASLEO. The imaging data for this survey were taken over the course of several observing runs from 2013 to 2015 , making a total of 7 nights at CASLEO and 30 at EABA. The main features of the observational systems are described in Table 1. The transmission curves of the filters used are shown in Fig. 1.

In EABA, series of 20 consecutive six-minute exposures were obtained for most of the PNe. On the other hand, the lower brightness objects were observed at CASLEO where, in general, three exposures of ten minutes each were acquired. Some objects, such as He 2-15, were purposely oversaturated to show 
Table 1. Summary of the main features of the observational systems.

\begin{tabular}{l|c|c}
\hline \hline & EABA & CASLEO \\
\hline Telescope size [m] & 1.54 & 2.15 \\
f-ratio & 4.9 & 8.6 \\
CCD brand & Apogee & Roper \\
Detector size [px] & $3072 \times 2048$ & $2048 \times 2048$ \\
Binning [px] & $3 \times 3$ & $5 \times 5$ \\
Pixel scale [arcsec/px] & 0.74 & 0.75 \\
Field of view [arcmin] & $12 \times 8$ & $5.1 \times 5.1$ \\
Central $\lambda\left[\AA{ }^{a}\right.$ & 6578 & 6582 \\
Bandpass filter [̊] ${ }^{a}$ & 20 & 18 \\
Filter transmission $T_{\lambda 6585}$ & 0.92 & 0.53 \\
Zero-point $k_{0}[\mathrm{mag}]$ & $-3.38 \pm 0.10$ & $-1.77 \pm 0.03$ \\
Ext. coeff. $k_{1}[\mathrm{mag} /$ airmass] & $-0.08 \pm 0.04$ & $-0.11 \pm 0.02$ \\
Lim. magnitude [mag arcmin ${ }^{-2}$ ] & 12.1 & 12.6 \\
\hline
\end{tabular}

Notes. The default gain for each instrument was used in all runs. ${ }^{(a)}$ See Fig. 1.

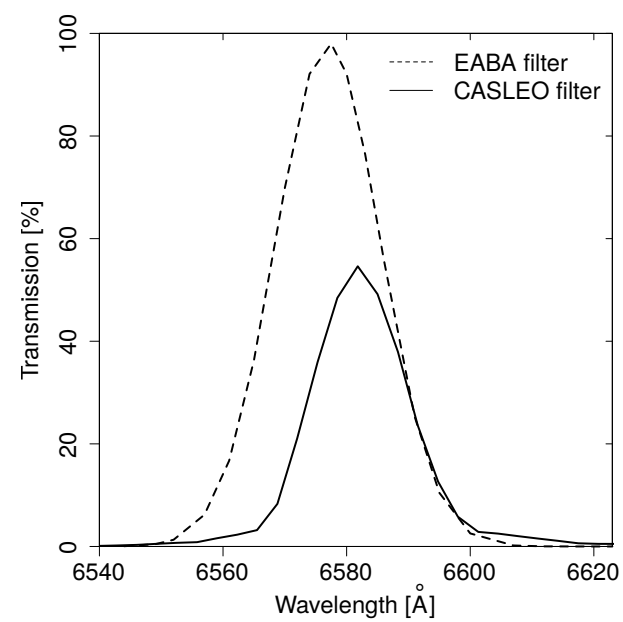

Fig. 1. Effective transmission curves for the filters (at 20 degrees Celsius).

the faintest regions of the nebula. Table 4 summarizes our sample of objects, all of which were observed at low zenith distance $\left(z<40^{\circ}\right)$.

Image reduction was performed using standard procedures with IRAF ${ }^{1}$. Thus, the data were first trimmed, bias-subtracted using a series of ten averaged bias frames and dark-subtracted (only in frames of EABA). The data were then flatfielded using a series of twilight flat fields. After the reduction was complete, the individual frames were spatially aligned to a common system using the IRAF task imalign. Typically, four stars were used to obtain this transformation. After that, all images were combined, cosmic rays removed, and the signal-to-noise $(\mathrm{S} / \mathrm{N})$ improved (IRAF task imcombine). Finally the images were rotated so that north was up and east to the left (IRAF tasks imtranspose and rotate).

The sky conditions during each night varied greatly. The combined effects of occasional poor telescope guiding and

1 IRAF: the Image Reduction and Analysis Facility is distributed by the National Optical Astronomy Observatories, which is operated by the Association of Universities for Research in Astronomy, Inc. (AURA) under cooperative agreement with the National Science Foundation (NSF).

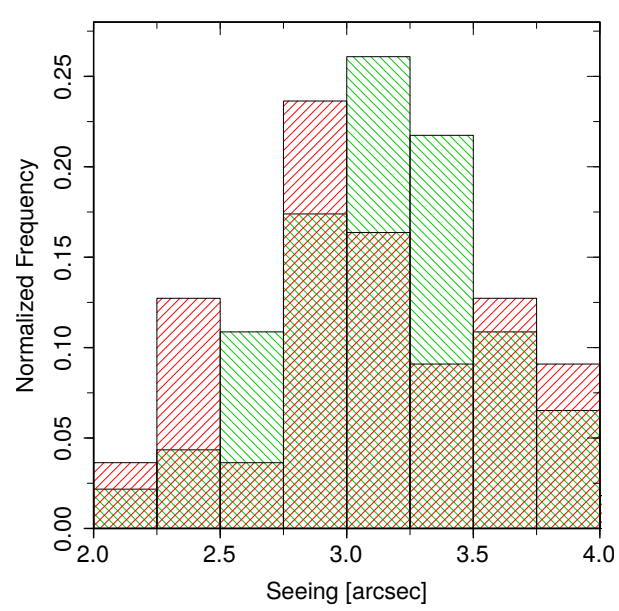

Fig. 2. FWHM of stars in CASLEO images, measured in the final combined frame (green) and individual reduced images (red).

variable dome and sky seeing resulted in the stellar full width at half maximum (FWHM) ranging from $2^{\prime \prime}$ to $5^{\prime \prime}$, as illustrated in Fig. 2 for CASLEO.

Moreover, since the final images suffered a slight degradation in quality due to the spatial alignment process, this effect is shown in Fig. 2. The combined images with a seeing greater than $4^{\prime \prime}$ were rejected. All the objects in the atlas have a $S / N>4$. More details about the images that were obtained at EABA are reported in Vena Valdarenas et al. (2015).

\subsection{Photometric calibration and detection limit}

To assess the detection limit at each observatory, two additional nights were devoted to the observation of two planetary nebulae and of a number of spectrophotometric standard stars to first derive the extinction coefficients for the filters and the sensibility of the instrumental systems. The observed nebulae are i) the faint PN G242.5-05.9 (on the night of 15-16 October 2015 from CASLEO); and ii) the brighter PN G002.7-52.4 (IC 5148, on the night of 17-18 October 2015 from EABA). During each night, several secondary and tertiary spectrophotometric standard stars from Hamuy et al. (1994) were also observed at airmasses in the ranges 1.01-1.88 (CASLEO) and 1.01-1.41 (EABA). Exposures for the stars reach from a few to $180 \mathrm{~s}$. Several images of $600 \mathrm{~s}$ of PN G242.5-05.9 at airmasses of $\sim 1.01$, and of $360 \mathrm{~s}$ at $\sim 1.15$ for PN G002.7-52.4 were obtained and combined to improve the $\mathrm{S} / \mathrm{N}$ relation.

Aperture photometry was performed on nebulae and stars with the package DAOPHOT in IRAF. For standard stars, apertures with radii of four times the FWHM of the stellar images were adopted without recourse to aperture correction. For PN G242.5-05.9 an aperture of 30 arcsec of radius centred on $\alpha=07^{\mathrm{h}} 26^{\mathrm{m}} 04.9, \delta=-28^{\circ} 58^{\prime} 23^{\prime \prime} \cdot 6(\mathrm{~J} 2000.0)$ was used, and for PN G002.7-52.4 an aperture of 79 arcsec of radius centred on $\alpha=21^{\mathrm{h}} 59^{\mathrm{m}} 35 \mathrm{~s}$. $1, \delta=-39^{\circ} 23^{\prime} 07^{\prime \prime}$. 1 (J2000.0) was chosen. In the first case, four stars that appear inside the nebula and a fifth one very near its apparent limits were successfully subtracted before performing the photometry. No star was subtracted from IC 5148.

The transformation equations are of the form:

$M(\lambda 6585)=m(\lambda 6585)+k_{0}+k_{1} \times X$,

where $M(\lambda 6585)$ is the calibrated monochromatic magnitude at $\lambda 6585, m(\lambda 6585)$ is the instrumental magnitude with an arbitrary 
Table 2. Adopted magnitudes at $\lambda 6585$ of spectrophotometric standards.

\begin{tabular}{lc}
\hline \hline Star & $M(\lambda 6585)$ \\
\hline EG 21 & 11.936 \\
HR 718 & 4.611 \\
HR 1544 & 4.634 \\
HR 9087 & 5.414 \\
LTT 377 & 11.106 \\
LTT 1020 & 11.320 \\
LTT 2415 & 12.125 \\
\hline
\end{tabular}

zero point of $21.0, k_{0}$ and $k_{1}$ are the zero-point and extinction coefficients, and $X$ is the airmass. It is worth mentioning that the system sensitivity, including the effects of telescope optics and detector response, and the filter transmission at $\lambda 6585\left(T_{\lambda 6585}\right.$, listed in Table 3), are included in $k_{0}$. The values of $M(\lambda 6585)$ for the observed spectrophotometric standards were obtained by interpolating in Tables 6 and 7 of Hamuy et al. (1994) and are listed in Table 2. The derived coefficients and their errors, as given by DAOPHOT, are given in Table 1. The monochromatic magnitudes of the nebulae are shown in Table 3; these are related to the flux density $f_{v}$ at $\lambda 6585$ by

$M(\lambda 6585)=-2.5 \times \log _{10}\left(f_{v}\right)-48.590$,

(Hamuy et al. 1994), where $f_{v}$ is expressed in $\operatorname{erg~cm}^{-2} \mathrm{~s}^{-1} \mathrm{~Hz}^{-1}$ This flux density has to be multiplied by the factor $c /(6585)^{2}$ ( $c$ is the speed of light) to give it as $f_{\lambda}$, that is, in the units $\operatorname{erg} \mathrm{cm}^{-2} \mathrm{~s}^{-1} \AA^{-1}$. After propagating the errors adequately, the flux densities of the nebulae and their logarithms are those listed in Table 3.

The surface brightnesses were calculated by adopting for circular shapes and areas of $0.217 \operatorname{arcmin}^{2}$ (PN G242.5-05.9) and $4.313 \operatorname{arcmin}^{2}$ (PN G002.7-52.4) for the nebulae. For the first, a surface brightness of $12.56 \mathrm{mag} \operatorname{arcmin}^{-2}$ at a $S / N \sim 4$ for an exposure time of $600 \mathrm{~s}$ is estimated, while for IC 5148 the value is $10.36 \mathrm{mag} \operatorname{arcmin}^{-2}$ with $S / N \sim 20$ and $360 \mathrm{~s}$. These brightnesses, expressed in magnitudes per squared arcmin and also in erg $\mathrm{cm}^{2} \mathrm{~s}^{-1} \AA^{-1} \operatorname{arcsec}^{-2}$, are listed in Table 3. While the surface brightness of the faint nebula observed at CASLEO can be adopted as the limiting magnitude for the images taken there, IC 5148 is rather bright, therefore we divided its flux by 5 to obtain a $\mathrm{S} / \mathrm{N}$ relation of $\sim 4$ like that measured in a combination of 360-s exposures of the faint nebula Hf 39 (Fig. B.11). The magnitude calculated with this new flux, $12.1 \mathrm{mag} \operatorname{arcmin}^{-2}$, can now be considered as the limiting magnitude in the images obtained at EABA for this survey.

It must be pointed out, finally, that the transformation derived in this section is in general not applicable to our collection of images, since observing on photometric nights was not a requirement for the original purposes of this work.

\section{Considerations about the survey}

\subsection{Narrow-band filters}

When interference filters are used in a converging beam, the primary effect is a shift of the transmittance peak towards shorter wavelengths (Eather \& Reasoner 1969).

Another important phenomenon that affects our observations is the bandpass shift due to variations of the ambient temperature (Pogge 1992). It moves towards the red or the blue, depending
Table 3. Calibrated magnitudes and fluxes at $\lambda 6585$ of two planetary nebulae.

\begin{tabular}{l|c|c}
\hline \hline & $\begin{array}{c}\text { PN G002.7-52.4 } \\
\text { (EABA) }\end{array}$ & $\begin{array}{c}\text { PN G242.5-05.9 } \\
\text { (CASLEO) }\end{array}$ \\
\hline$M(\lambda 6585)$ & $\begin{array}{c}8.78 \pm 0.04 \\
f_{\lambda}{ }^{2}\end{array}$ & $14.20 \pm 0.12$ \\
$\log f_{\lambda}$ & $(7.8 \pm 0.3) \times 10^{-13}$ & $(5.2 \pm 0.6) \times 10^{-15}$ \\
Surface brightness $^{b}$ & $12.11 \pm 0.04$ & $-14.3 \pm 0.1$ \\
Surface brightness $^{c}$ & $(5.0 \pm 0.36 \pm 0.05$ & $12.56 \pm 0.14$ \\
\hline
\end{tabular}

Notes. ${ }^{(a)} \operatorname{erg~cm}{ }^{-2} \mathrm{~s}^{-1} \AA^{-1}$. ${ }^{(b)}$ mag $\operatorname{arcmin}^{-2}$. (c) $\operatorname{erg~cm}{ }^{-2} \mathrm{~s}^{-1} \AA^{-1} \operatorname{arcsec}^{-2}$.

on whether the temperature increases or decreases, respectively, following a linear temperature dependence. For a narrow-band filter, the shift in mean wavelength due to temperature is about $0.18 \AA$ per degree Celsius. An additional problem is posed by the age of filters: after 6-10 yr they usually deteriorate, mainly losing transmittance.

On the other hand, we have to take into account the radial velocity of the objects. The radial velocity of a PN could cause the [N II] emission to fall outside of the effective bandpass of the filter. Unfortunately, many objects included in our atlas do not have radial velocity determinations, in particular the objects extracted from the MASH catalogue (Parker et al. 2005).

The filter peak transmittance was determined at a temperature of $20^{\circ} \mathrm{C}$, which means that those objects that were observed from EABA in winter (where the temperature may drop to $5^{\circ}$ ) and with positive radial velocity might be contaminated by $\mathrm{H} \alpha$ emission. These objects are labelled in Table 4, where we used the radial velocity data from Durand et al. (1998) as a reference.

Finally, it is necessary to consider another possible nebular emission in this wavelength range. Fortunately, there are only very few nebular lines (C II at 6578.05 and $6582.88 \AA$ ) that could contaminate our data. Nevertheless, contamination by continuous nebular emission will always be present.

\subsection{Comparison with other observations}

Our work has objects in common with the catalogues of Balick (1987) and Hua et al. (1998). These objects are labelled in Table 4. With the former, the only nebula in common is NGC 2610, which we observed from EABA. In this case the Balick images are deeper.

Our atlas includes ten PNe that were observed by Hua et al. (1998). The comparison in this case is more difficult, since Hua used three different telescopes and we used two. However, the images of those PNe that were observed from CASLEO are of quality and depth comparable to those obtained by Hua et al. (1998) (e.g. He 2-70 and K 1-3).

\section{Results}

The atlas of monochromatic images is presented in Figs. B.1 to B.18. In all the images we tried different brightness and contrast levels to emphasize the most interesting features of each object. A logarithmic scale is indicated with an "(L)" in the caption of the figures.

Our atlas includes objects that do not have any morphological classification (e.g. Wray 16-20, ESO 259-10, LoTr 7, and VBRC 6) or are very poorly studied (e.g. HaWe 9, SaWe 1, 
Table 4. PNe included in our atlas.

\begin{tabular}{|c|c|c|c|c|c|c|c|}
\hline$N$ & Name & PN G & RA (J2000) & $\operatorname{Dec}(\mathrm{J} 2000)$ & Telescope & Images & Figure \\
\hline 1 & IC $5148-50^{*, \dagger}$ & $002.7-52.4$ & 215931.7 & -392236.2 & $\mathrm{E}$ & 10 & B.1 \\
\hline 2 & $\mathrm{Hb} 4$ & $003.1+02.9$ & 174152.7 & -244208.0 & $\mathrm{E}$ & 11 & B. 1 \\
\hline 3 & SaWe $3^{*, \dagger}$ & $013.8-02.8$ & 182603.1 & -181205.6 & $\mathrm{E}$ & 15 & B. 1 \\
\hline 4 & HDW 12 & $014.8-25.6$ & 195813.2 & -262815.4 & $\mathrm{C}$ & 3 & B. 1 \\
\hline 5 & DeHt 3 & $019.4-13.6$ & 191704.6 & -180134.2 & $\mathrm{E}$ & 17 & B. 1 \\
\hline 6 & A 66 & $019.8-23.7$ & 195731.8 & -213636.8 & $\mathrm{C}$ & 8 & B. 1 \\
\hline 7 & A 67 & $043.5-13.4$ & 195829.3 & +030222.6 & $\mathrm{C}$ & 3 & B. 2 \\
\hline 8 & NGC $246^{*}$ & $118.8-74.7$ & 004703.8 & -115221.6 & $\mathrm{C}$ & 3 & B. 2 \\
\hline 9 & PHR0615-0025 & $209.1-08.2$ & 061520.4 & -002549.1 & $\mathrm{C}$ & 3 & B. 2 \\
\hline 10 & $\mathrm{~K} 1-11$ & $215.6+11.1$ & 073607.7 & +024217.2 & $\mathrm{C}$ & 8 & B. 2 \\
\hline 11 & PHR0702-0324 & $217.2+00.9$ & 070234.2 & -032434.9 & $\mathrm{C}$ & 3 & B. 2 \\
\hline 12 & PHR0705-0924 & $222.9-01.1$ & 070551.4 & -092411.2 & $\mathrm{C}$ & 4 & B. 2 \\
\hline 13 & PHR0700-1143 & $224.3-03.4$ & 070005.8 & -114350.9 & $\mathrm{C}$ & 5 & B. 3 \\
\hline 14 & PHR0716-1053 & $225.4+00.4$ & 071608.0 & -105306.0 & $\mathrm{C}$ & 6 & B. 3 \\
\hline 15 & PHR0711-1238 & $226.4-01.3$ & 071143.3 & -123803.1 & $\mathrm{C}$ & 6 & B. 3 \\
\hline 16 & M 1-16 & $226.7+05.6$ & 073718.9 & -093848.1 & $\mathrm{E}$ & 10 & B. 3 \\
\hline 17 & PHR0719-1222 & $227.1+00.5$ & 071946.7 & -122246.9 & $\mathrm{C}$ & 3 & B. 3 \\
\hline 18 & PHR0727-1259 & $228.6+01.9$ & 072749.0 & -125930.1 & $\mathrm{C}$ & 3 & B. 3 \\
\hline 19 & PHR0727-1707 & $232.1-00.1$ & 072708.3 & -170714.2 & $\mathrm{C}$ & 5 & B. 4 \\
\hline 20 & PHR0724-1757 & $232.6-01.0$ & 072443.4 & -175751.1 & $\mathrm{C}$ & 3 & B. 4 \\
\hline 21 & SaWe 1 & $233.0-10.1$ & 065041.0 & -222609.3 & $\mathrm{C}$ & 3 & B. 4 \\
\hline 22 & HaWe 9 & $236.0-10.6$ & 065429.0 & -252111.6 & $\mathrm{C}$ & 6 & B. 4 \\
\hline 23 & PHR0730-2151 & $236.7-01.6$ & 073046.9 & -215143.9 & $\mathrm{C}$ & 3 & B. 4 \\
\hline 24 & ESO 427-19 & $239.6-12.0$ & 065512.1 & -290728.4 & $\mathrm{C}$ & 3 & B.4 \\
\hline 25 & NGC $2610^{\dagger}$ & $239.6+13.9$ & 083323.5 & -160855.9 & $\mathrm{E}$ & 13 & B.5 \\
\hline 26 & PHR0726-2858 & $242.5-05.9$ & 072604.8 & -285823.5 & $\mathrm{C}$ & 4 & B. 5 \\
\hline 27 & PRTM 1 & $243.8-37.1$ & 050301.7 & -394544.0 & $\mathrm{C}$ & 3 & B.5 \\
\hline 28 & PHR0742-3247 & $247.5-04.7$ & 074223.6 & -324744.9 & $\mathrm{C}$ & 3 & B.5 \\
\hline 29 & A 23 & $249.3-05.4$ & 074318.9 & -344513.0 & $\mathrm{C}$ & 3 & B.5 \\
\hline 30 & PHR0834-2819 & $249.8+07.1$ & 083418.1 & -281903.0 & $\mathrm{C}$ & 3 & B.5 \\
\hline 31 & PHR0803-3331 & $250.4-01.3$ & 080312.5 & -333101.9 & $\mathrm{C}$ & 4 & B.6 \\
\hline 32 & PHR0736-3901 & $252.4-08.7$ & 073625.2 & -390131.1 & $\mathrm{C}$ & 3 & B. 6 \\
\hline 33 & PHR0820-3516 & $253.9+00.7$ & 082052.4 & -351632.9 & $\mathrm{C}$ & 3 & B. 6 \\
\hline 34 & VBRC 1 & $257.5+00.6$ & 083058.0 & -381952.3 & $\mathrm{C}$ & 3 & B. 6 \\
\hline 35 & Wray 17-1 & $258.0-15.7$ & 071448.0 & -465724.4 & $\mathrm{C}$ & 3 & B.6 \\
\hline 36 & He $2-11$ & $259.1+00.9$ & 083708.2 & -392625.5 & $\mathrm{C}$ & 3 & B.6 \\
\hline 37 & Wray $16-20$ & $260.7-03.3$ & 082340.4 & $-43 \quad 1244.0$ & $\mathrm{C}$ & 3 & B. 7 \\
\hline 38 & He $2-15$ & $261.6+03.0$ & 085330.6 & -400334.4 & $\mathrm{C}$ & 3 & B.7 \\
\hline 39 & NGC 2818 & $261.9+08.5$ & 091600.5 & -363731.6 & $\mathrm{E}$ & 20 & B.7 \\
\hline 40 & Wray $17-18$ & $262.6-04.6$ & 082353.7 & -453109.9 & $\mathrm{E}$ & 10 & B.7 \\
\hline 41 & ESO 209-15 & $263.3-08.8$ & 080510.9 & -482330.1 & $\mathrm{E}$ & 20 & B.7 \\
\hline 42 & He $2-7^{\dagger}$ & $264.1-08.1$ & 081131.9 & -484314.5 & $\mathrm{C}$ & 3 & B.7 \\
\hline 43 & ESO 259-10 & $265.1-04.2$ & 083407.0 & -471638.1 & $\mathrm{C}$ & 3 & B. 8 \\
\hline 44 & PHR0911-4205 & $265.4+04.2$ & 091148.5 & -420509.9 & $\mathrm{C}$ & 4 & B. 8 \\
\hline 45 & PHR0927-4347 & $268.6+05.0$ & 092728.1 & -434753.2 & $\mathrm{C}$ & 6 & B. 8 \\
\hline 46 & PHR0905-4753 & $268.9-00.4$ & 090539.6 & -475338.0 & $\mathrm{C}$ & 3 & B. 8 \\
\hline 47 & He $2-37$ & $274.6+03.5$ & 094724.3 & -485819.1 & $\mathrm{C}$ & 3 & B. 8 \\
\hline 48 & PHR0924-5506 & $276.1-03.3$ & 092415.1 & -550624.8 & $\mathrm{C}$ & 6 & B. 8 \\
\hline 49 & NGC 2899 & $277.1-03.8$ & 092703.5 & -560618.3 & $\mathrm{E}$ & 20 & B.9 \\
\hline 50 & PHR0958-5039 & $277.1+03.3$ & 095810.2 & -503934.9 & $\mathrm{C}$ & 6 & B.9 \\
\hline 51 & Wray $17-31$ & $277.7-03.5$ & 093127.1 & -561741.1 & $\mathrm{E}$ & 20 & B.9 \\
\hline 52 & He $2-32$ & $278.5-04.5$ & 093055.9 & -573657.8 & $\mathrm{C}$ & 3 & B.9 \\
\hline 53 & PHR0940-5658 & $279.1-03.1$ & 094052.5 & -565800.1 & $\mathrm{C}$ & 3 & B.9 \\
\hline 54 & PHR1010-5146 & $279.2+03.5$ & 101004.1 & -514648.0 & $\mathrm{C}$ & 6 & B.9 \\
\hline 55 & He $2-36$ & $279.6-03.1$ & 094326.0 & -571659.7 & $\mathrm{E}$ & 10 & B. 10 \\
\hline
\end{tabular}

Notes. The PNe are denoted by their common name and by their PN G designation. Sixth column lists the telescope used in each observation, EABA and CASLEO, indicated by "E" and "C" respectively. Seventh column lists the number of combined images and eighth column shows the figure number for each nebulae. PNe observed by Hua et al. (1998) and Balick (1987) are marked by “*” and objects labelled with “(i)” have probably $\mathrm{H} \alpha$ contamination or only the continuum emission is detected. 
W. A. Weidmann et al.: Atlas of monochromatic images of planetary nebulae $(R N)$

Table 4. continued.

\begin{tabular}{|c|c|c|c|c|c|c|c|}
\hline$N$ & Name & PN G & RA (J2000) & $\operatorname{Dec}(\mathrm{J} 2000)$ & Telescope & Images & Figure \\
\hline 56 & Ste $2-1$ & $280.0+02.9$ & 101157.9 & -523815.2 & $\mathrm{C}$ & 3 & B. 10 \\
\hline 57 & PHR1019-6059 & $285.5-03.3$ & 101927.6 & -605909.9 & $\mathrm{C}$ & 3 & B. 10 \\
\hline 58 & PHR1036-5909 & $286.3-00.7$ & 103609.2 & -590920.2 & $\mathrm{C}$ & 6 & B. 10 \\
\hline 59 & He $2-55$ & $286.3+02.8$ & 104843.1 & -560323.0 & $\mathrm{E}$ & 14 & B. 10 \\
\hline 60 & PHR1046-6109 & $288.4-01.8$ & 104651.1 & -610923.0 & $\mathrm{C}$ & 3 & B. 10 \\
\hline 61 & Hf 38 & $288.4+00.3$ & 105434.9 & -590948.7 & $\mathrm{C}$ & 3 & B. 11 \\
\hline 62 & PHR1058-5853 & $288.7+00.8$ & 105802.9 & -585334.1 & $\mathrm{C}$ & 3 & B. 11 \\
\hline 63 & Hf 39 & $288.9-00.8$ & 105358.9 & -602642.0 & $\mathrm{E}$ & 3 & B. 11 \\
\hline 64 & Hf $48^{\dagger}$ & $290.1-00.4$ & 110355.6 & -603605.6 & $\mathrm{E}$ & 20 & B. 11 \\
\hline 65 & Fg 1 & $290.5+07.9$ & 112835.9 & -525606.4 & $\mathrm{E}$ & 10 & B. 11 \\
\hline 66 & NGC $3699^{\dagger}$ & $292.6+01.2$ & 112759.2 & -595732.0 & $\mathrm{C}$ & 3 & B. 11 \\
\hline 67 & He $2-70^{\dagger}$ & $293.6+01.2$ & 113512.6 & -601654.1 & $\mathrm{C}$ & 3 & B. 12 \\
\hline 68 & $\mathrm{BlDz} 1$ & $293.6+10.9$ & 115303.8 & -505041.8 & $\mathrm{E}$ & 25 & B. 12 \\
\hline 69 & NGC 3918 & $294.6+04.7$ & 115018.9 & -571051.4 & $\mathrm{E}$ & 20 & B. 12 \\
\hline 70 & He $2-72^{\dagger}$ & $294.9-00.6$ & 114137.9 & -622856.9 & $\mathrm{C}$ & 3 & B. 12 \\
\hline 71 & NGC 3195 & $296.6-20.0$ & 100922.0 & -805130.6 & $\mathrm{E}$ & 10 & B. 12 \\
\hline 72 & He $2-76$ & $298.2-01.7$ & 120826.0 & -641212.1 & $\mathrm{E}$ & 13 & B. 12 \\
\hline 73 & PHR1221-5907 & $299.0+03.5$ & 122100.7 & -590732.9 & $\mathrm{C}$ & 4 & B.13 \\
\hline 74 & $\mathrm{~K} 1-23$ & $299.0+18.4$ & 123052.0 & -441422.2 & $\mathrm{E}$ & 20 & B. 13 \\
\hline 75 & Wray $16-121$ & $302.6-00.9$ & 124831.1 & -634956.5 & $\mathrm{C}$ & 3 & B. 13 \\
\hline 76 & PHR1250-6346 & $302.7-00.9$ & 125004.4 & -634651.9 & $\mathrm{E}$ & 14 & B. 13 \\
\hline 77 & Wray $16-122$ & $304.2+05.9$ & 130041.4 & -565328.3 & $\mathrm{C}$ & 6 & B. 13 \\
\hline 78 & NGC $5189^{\dagger}$ & $307.2-03.4$ & 133341.9 & -655828.9 & $\mathrm{E}$ & 20 & B. 13 \\
\hline 79 & He 2-99 & $309.0-04.2$ & 135231.0 & -662328.0 & $\mathrm{E}$ & 19 & B. 14 \\
\hline 80 & VBRC 5 & $309.2+01.3$ & 134359.2 & -604940.4 & $\mathrm{C}$ & 3 & B. 14 \\
\hline 81 & $\mathrm{He} 2-103$ & $310.7-02.9$ & 140536.9 & -644057.0 & $\mathrm{E}$ & 20 & B. 14 \\
\hline 82 & LoTr 7 & $310.8-05.9$ & 141523.1 & -673157.6 & $\mathrm{E}$ & 14 & B. 14 \\
\hline 83 & PHR1408-6229 & $311.7-00.9$ & 140847.3 & -622957.8 & $\mathrm{C}$ & 3 & B. 14 \\
\hline 84 & He $2-111$ & $315.0-00.3$ & 143318.3 & -604944.6 & $\mathrm{E}$ & 14 & B. 14 \\
\hline 85 & He $2-119^{\dagger}$ & $317.1-05.7$ & 151039.9 & -644019.1 & $\mathrm{E}$ & 20 & B. 15 \\
\hline 86 & VBRC 6 & $317.8+03.3$ & 144135.7 & -561506.6 & $\mathrm{E}$ & 25 & B. 15 \\
\hline 87 & He $2-114$ & $318.3-02.0$ & 150408.8 & -605321.2 & $\mathrm{E}$ & 20 & B. 15 \\
\hline 88 & He $2-116$ & $318.3-02.5$ & 150600.8 & -612124.4 & $\mathrm{E}$ & 10 & B. 15 \\
\hline 89 & ESO $135-04$ & $318.4-03.0$ & 150842.8 & -614403.9 & $\mathrm{E}$ & 20 & B. 15 \\
\hline 90 & IC $4406^{\dagger}$ & $319.6+15.7$ & 142226.5 & -440906.0 & $\mathrm{E}$ & 10 & B. 15 \\
\hline 91 & He $2-120$ & $321.8+01.9$ & 151156.1 & -553951.1 & $\mathrm{E}$ & 10 & B. 16 \\
\hline 92 & Mz 1 & $322.4-02.6$ & 153416.7 & -590859.5 & $\mathrm{E}$ & 10 & B. 16 \\
\hline 93 & He $2-163$ & $327.8-07.2$ & 162930.3 & -590922.3 & $\mathrm{E}$ & 12 & B. 16 \\
\hline 94 & He $2-146$ & $328.9-02.4$ & 161040.9 & -545731.9 & $\mathrm{E}$ & 20 & B. 16 \\
\hline 95 & Mz 2 & $329.3-02.8$ & 161432.1 & -545704.0 & $\mathrm{E}$ & 20 & B. 16 \\
\hline 96 & PHR1557-5128 & $329.7+01.4$ & 155707.4 & -512800.8 & $\mathrm{E}$ & 20 & B. 16 \\
\hline 97 & He $2-165$ & $331.5-03.9$ & 162959.6 & -540936.7 & $\mathrm{E}$ & 12 & B. 17 \\
\hline 98 & He $2-164$ & $332.0-03.3$ & 162953.2 & -532304.1 & $\mathrm{E}$ & 14 & B. 17 \\
\hline 99 & IC 4642 & $334.3-09.3$ & 171145.4 & -552402.4 & $\mathrm{E}$ & 10 & B. 17 \\
\hline 100 & Lo 12 & $340.8+10.8$ & 160826.3 & -370848.3 & $\mathrm{E}$ & 20 & B. 17 \\
\hline 101 & NGC 6153 & $341.8+05.4$ & 163130.9 & -401522.5 & $\mathrm{E}$ & 14 & B. 17 \\
\hline 102 & H $1-3$ & $342.7+00.7$ & 165331.5 & -423918.1 & $\mathrm{E}$ & 10 & B. 17 \\
\hline 103 & Pe $1-8$ & $342.9-02.0$ & 170622.8 & -441312.1 & $\mathrm{E}$ & 10 & B. 18 \\
\hline 104 & He 2-207 & $342.9-04.9$ & 171932.5 & -455310.0 & $\mathrm{E}$ & 18 & B. 18 \\
\hline 105 & IC 4637 & $345.4+00.1$ & 170509.0 & -405257.1 & $\mathrm{E}$ & 10 & B. 18 \\
\hline 106 & K $1-3^{\dagger}$ & $346.9+12.4$ & 162317.3 & -314456.9 & $\mathrm{E}$ & 12 & B. 18 \\
\hline 107 & K $2-16^{\dagger}$ & $352.9+11.4$ & 164449.1 & -280405.4 & $\mathrm{E}$ & 17 & B. 18 \\
\hline 108 & Wray $16-411$ & $353.7-12.8$ & 182641.5 & -402951.1 & $\mathrm{E}$ & 14 & B. 18 \\
\hline
\end{tabular}

ESO 427-19, and ESO 209-15); in particular, it contains images of recently classified PNe (Parker et al. 2005). Moreover, this catalogue shows and reveals very interesting structures in several nebulae. For example, PHR0716-1053 displays a non-concentric multishell, resembling the mysterious rings of supernova 1987A, or the outstanding planetary nebulae VBRC 1 and Wray 16-121 that shows a capricious distribution of ionized material. 
We compared our images with those available in the literature (even if they were taken in a different filter), to compare our classification morphology with previous ones given by other authors. As a result, we reclassified some objects and provided one to those nebulae that did not have previous classification.

We hope that these new images and morphological descriptions presented here will provide a guide for future research, thus contributing to a better understanding of the final stages of stellar evolution.

Acknowledgements. We thank the referee, Romano Corradi, whose very useful remarks helped us to improve this paper. This work is partially supported by CONICET (PIP 11220120100298). The CCD and data acquisition system at CASLEO has been financed by R. M. Rich through US NSF grant AST-9015827. This work is partially based on observations obtained with the $1.54 \mathrm{~m}$ telescope of the Estación Astrofísica de Bosque Alegre, dependent on the Universidad Nacional de Córdoba, Argentina. This research has made use of SAO Image DS9, developed by Smithsonian Astrophysical Observatory. This research made use of the SIMBAD database, operated at the CDS, Strasbourg, France.

\section{References}

Acker, A., Marcout, J., Ochsenbein, F., et al. 1992, The Strasbourg-ESO Catalogue of Galactic Planetary Nebulae. Parts I, II. (ESO Publ.)

Ali, A., El-Nawawy, M. S., \& Pfleiderer, J. 2000, Ap\&SS, 271, 245

Ali, A., Sabin, L., Snaid, S., \& Basurah, H. M. 2012, A\&A, 541, A98

Balick, B. 1987, AJ, 94, 671

Balick, B. 1989, in Planetary Nebulae, ed. S. Torres-Peimbert, IAU Symp., 131, 83

Boffin, H. M. J., Miszalski, B., \& Jones, D. 2012a, A\&A, 545, A146

Boffin, H. M. J., Miszalski, B., Rauch, T., et al. 2012b, Science, 338, 773

Bohigas, J. 2003, Rev. Mex. Astron. Astrofis., 39, 149

Chu, Y.-H., Jacoby, G. H., \& Arendt, R. 1987, ApJS, 64, 529

Chu, Y.-H., Gruendl, R. A., Guerrero, M. A., et al. 2009, AJ, 138, 691

Corradi, R. L. M., \& Schwarz, H. E. 1993a, A\&A, 278, 247

Corradi, R. L. M., \& Schwarz, H. E. 1993b, A\&A, 273, 247

Corradi, R. L. M., \& Schwarz, H. E. 1995, A\&A, 293, 871

Corradi, R. L. M., Manso, R., Mampaso, A., \& Schwarz, H. E. 1996, A\&A, 313, 913

Corradi, R. L. M., Perinotto, M., Villaver, E., Mampaso, A., \& Gonçalves, D. R. 1999, ApJ, 523, 721

Corradi, R. L. M., Schönberner, D., Steffen, M., \& Perinotto, M. 2003, MNRAS, 340,417

Corradi, R. L. M., Sánchez-Blázquez, P., Mellema, G., Giammanco, C., \& Schwarz, H. E. 2004, A\&A, 417, 637

Drew, J. E., Gonzalez-Solares, E., Greimel, R., et al. 2014, MNRAS, 440, 2036 Dufour, R. J. 1984, ApJ, 287, 341

Durand, S., Acker, A., \& Zijlstra, A. 1998, A\&AS, 132, 13

Eather, R. H., \& Reasoner, D. L. 1969, Appl. Opt., 8, 227

Fredrick, L. W., \& West, R. M. 1984, A\&AS, 56, 325

Frew, D. J., Bojičić, I. S., Parker, Q. A., et al. 2014, MNRAS, 440, 1080

Gonçalves, D. R., Corradi, R. L. M., \& Mampaso, A. 2002, in Rev. Mex. Astron. Astrofis. Conf. Ser. 13, eds. W. J. Henney, W. Steffen, L. Binette, \& A. Raga, 119

Gorny, S. K., Stasińska, G., \& Tylenda, R. 1997, A\&A, 318, 256

Górny, S. K., Schwarz, H. E., Corradi, R. L. M., \& Van Winckel, H. 1999, A\&AS, 136, 145

Goudis, G., \& Meaburn, J. 1978, A\&A, 62, 283

Greve, A., \& van Genderen, A. M. 1987, A\&A, 174, 243

Guerrero, M. A., Toalá, J. A., Medina, J. J., et al. 2013, A\&A, 557, A121

Hajian, A. R., Balick, B., Terzian, Y., \& Perinotto, M. 1997, ApJ, 487, 304

Hajian, A. R., Movit, S. M., Trofimov, D., et al. 2007, ApJS, 169, 289

Hamuy, M., Suntzeff, N. B., Heathcote, S. R., et al. 1994, PASP, 106, 566

Hartl, H., \& Weinberger, R. 1987, A\&AS, 69, 519

Hoogerwerf, R., Szentgyorgyi, A., Raymond, J., et al. 2007, ApJ, 670, 442

Hua, C. T., Dopita, M. A., \& Martinis, J. 1998, A\&AS, 133, 361

Huggins, P. J., Forveille, T., Bachiller, R., \& Cox, P. 2000, ApJ, 544, 889

Jones, D., Boffin, H. M. J., Miszalski, B., et al. 2014, A\&A, 562, A89

Kaler, J. B., Shaw, R. A., Feibelman, W. A., \& Lutz, J. H. 1989, ApJS, 70, 213
Kerber, F., Furlan, E., Roth, M., Galaz, G., \& Chanamé, J. C. 2000, PASP, 112, 542

Lee, T.-H., Lim, J., \& Kwok, S. 2007a, ApJ, 665, 341

Lee, T.-H., Stanghellini, L., Ferrario, L., \& Wickramasinghe, D. 2007b, AJ, 133, 987

Liu, X.-W., Storey, P. J., Barlow, M. J., et al. 2000, MNRAS, 312, 585

Longmore, A. J., \& Tritton, S. B. 1980, MNRAS, 193, 521

López, J. A. 2000, in Rev. Mex. Astron. Astrofis. Conf. Ser. 9, eds. S. J. Arthur, N. S. Brickhouse, \& J. Franco, 201

López, J. A., Falcón, L. H., Ruiz, M. T., \& Roth, M. 1989, in Planetary Nebulae, ed. S. Torres-Peimbert, IAU Symp., 131, 179

Lopez, J. A., Falcon, L. H., Ruiz, M. T., \& Roth, M. 1991, A\&A, 241, 526

Lopez, J. A., Roth, M., \& Tapia, M. 1993, A\&A, 267, 194

López, J. A., Steffen, W., \& Meaburn, J. 1997, ApJ, 485, 697

Louise, R., Macron, A., Pascoli, G., \& Maurice, E. 1987, A\&AS, 70, 201

Manchado, A., Guerrero, M. A., Stanghellini, L., \& Serra-Ricart, M. 1996, The IAC morphological catalog of northern Galactic planetary nebulae (La Laguna, Spain: Instituto de Astrofisica de Canarias)

Marston, A. P., Bryce, M., Lopez, J. A., Palmer, J. W., \& Meaburn, J. 1998, A\&A, 329, 683

Meaburn, J., \& Walsh, J. R. 1989, A\&A, 223, 277

Miranda, L. F., Pereira, C. B., \& Guerrero, M. A. 2009, AJ, 137, 4140

Miszalski, B., Acker, A., Parker, Q. A., \& Moffat, A. F. J. 2009, A\&A, 505, 249

Moore, S. L. 2007, J. Brit. Astron. Assoc., 117, 204

Moreno, H., Gutiérrez-Moreno, A., Torres, C., \& Wenderoth, E. 1987, Rev. Mex. Astron. Astrofis., 14, 520

Moreno, H., Gutierrez-Moreno, A., \& Torres, C. 1991, PASP, 103, 275

O’Dell, C. R., Balick, B., Hajian, A. R., Henney, W. J., \& Burkert, A. 2002, AJ, 123,3329

Palmer, J. W., Lopez, J. A., Meaburn, J., \& Lloyd, H. M. 1996, A\&A, 307, 225

Parker, Q. A., Phillipps, S., Pierce, M. J., et al. 2005, MNRAS, 362, 689

Parker, Q. A., Acker, A., Frew, D. J., et al. 2006, MNRAS, 373, 79

Pascoli, G. 1990, A\&A, 232, 184

Peimbert, M., \& Torres-Peimbert, S. 1983, in Planetary Nebulae, ed. D. R. Flower, IAU Symp., 103, 233

Pereyra, M., Richer, M. G., \& López, J. A. 2013, ApJ, 771, 114

Perinotto, M., \& Corradi, R. L. M. 1998, A\&A, 332, 721

Perinotto, M., Purgathofer, A., Pasquali, A., \& Patriarchi, P. 1994, A\&AS, 107, 481

Phillips, J. P. 2003, MNRAS, 344, 501

Phillips, J. P., \& Reay, N. K. 1983, A\&A, 117, 33

Pogge, R. W. 1992, in Astronomical CCD Observing and Reduction Techniques, ed. S. B. Howell, ASP Conf. Ser., 23, 195

Polcaro, V. F., Rossi, C., Viotti, R., \& Norci, L. 1997, A\&A, 318, 571

Pottasch, S. R., Mo, J.-E., \& Dennefeld, M. 1986, A\&A, 155, 397

Rauch, T., Köppen, J., Napiwotzki, R., \& Werner, K. 1999, A\&A, 347, 169

Reay, N. K., Atherton, P. D., \& Taylor, K. 1984, MNRAS, 206, 71

Richer, M. G., McCall, M. L., \& Martin, P. G. 1991, ApJ, 377, 210

Sabin, L., Vázquez, R., López, J. A., García-Díaz, M. T., \& Ramos-Larios, G. 2012, Rev. Mex. Astron. Astrofis., 48, 165

Saurer, W., \& Weinberger, R. 1987, A\&AS, 69, 527

Schwarz, H. E. 1992, A\&A, 264, L1

Schwarz, H. E., Corradi, R. L. M., \& Melnick, J. 1992, A\&AS, 96, 23

Smith, L. J., Crowther, P. A., \& Prinja, R. K. 1994, A\&A, 281, 833

Smith, N., Bally, J., \& Walawender, J. 2007, AJ, 134, 846

Stahl, O. 1987, A\&A, 182, 229

Stanghellini, L., Corradi, R. L. M., \& Schwarz, H. E. 1993a, A\&A, 279, 521

Stanghellini, L., Corradi, R. L. M., \& Schwarz, H. E. 1993b, A\&A, 276, 463

Streicher, M. 2010, Monthly Notes of the Astronomical Society of South Africa, 69, 229

Szentgyorgyi, A., Franco, J., López-Martín, L., Raymond, J. C., \& Villaver, E. 2003a, in Rev. Mex. Astron. Astrofis. Conf. Ser. 15, eds. J. Arthur, \& W. J. Henney, 84

Szentgyorgyi, A., Raymond, J., Franco, J., Villaver, E., \& López-Martín, L. 2003b, ApJ, 594, 874

Tweedy, R. W., \& Kwitter, K. B. 1996, ApJS, 107, 255

Vázquez, R. 2012, ApJ, 751, 116

Vena Valdarenas, R. R., Schmidt, E. O., Volpe, M. G., Weidmann, W., \& Mudrik, A. 2015, Boletin de la Asociacion Argentina de Astronomia (La Plata, Argentina), 57, 197

Walsh, A. J., Hyland, A. R., Robinson, G., \& Burton, M. G. 1997, MNRAS, 291, 261

Zhang, C. Y., \& Kwok, S. 1998, ApJS, 117, 341

Zuckerman, B., \& Aller, L. H. 1986, ApJ, 301, 772 


\section{Appendix A: Notes on individual objects}

PN G002.7-52.4: ring-like PN with an inner highly elongated elliptical ring with several irregularities in the brightness and an external round fainter nebulosity. More images can be found in Streicher (2010), Moreno et al. (1991), and Chu et al. (1987).

PN G003.1+02.9: Stanghellini et al. (1993a) classified this $\mathrm{PN}$ as irregular. We disagree with this classification because in our image we can see an elliptical structure with prominent jets. More images can be found in Miszalski et al. (2009), López (2000), Zhang \& Kwok (1998), Hajian et al. (1997), López et al. (1997), and Corradi et al. (1996).

PN G013.8-02.8: bipolar PN. More images can be found in Saurer \& Weinberger (1987).

PN G014.8-25.6: faint nebulosity with no obvious symmetry.

PN G019.4-13.6: middle elliptical according to the morphological scheme of Balick (1989). More images can be found in Bohigas (2003).

PN G043.5-13.4: PN with no clear symmetry, roughly elliptical with a nearly rectangular shape. More images can be found in Schwarz et al. (1992).

PN G118.8-74.7: a bright and well-studied object. Phillips (2003) classified this PN as round. Our image shows that it has an elliptical shape with an inner filamentary structure and intense patches to the SE. We suggest that the object needs to be reclassified. Images of this object are found in Pereyra et al. (2013), Chu et al. (2009), Hoogerwerf et al. (2007), Moore (2007), Szentgyorgyi et al. (2003b,a).

PN G209.1-08.2: in the MASH images the object is very faint and does not reveal any morphology. In our image it shows a clearly round morphology, with non-uniform brightness.

PN G215.6+11.1: curious morphology, nebula with a nearly rectangular shape. Possible bipolar PN.

PN G217.2+00.9: probable interaction with the ISM.

PN G224.3-03.4: probable interaction with the ISM.

PN G225.4+00.4: intriguing morphology, it displays a nonconcentric multishell, similar to the mysterious rings of supernova 1987A.

PN G226.7+05.6: all authors agree that the object shows a bipolar morphology. Stanghellini et al. (1993b) classified it as bipolar with an outer structure. More images can be found in Huggins et al. (2000), Perinotto \& Corradi (1998), Corradi \& Schwarz (1995), Schwarz (1992), Schwarz et al. (1992), and Corradi \& Schwarz (1993a).

PN G233.0-10.1: ring-like PN. More images can be found in Saurer \& Weinberger (1987).

PN G236.0-10.6: object poorly studied, morphology type one-side (see Ali et al. 2000) with radial filaments. More images can be found in Hartl \& Weinberger (1987).

PN G236.7-01.6: our image shows two areas of lower brightness that are not visible in the $\mathrm{H} \alpha$ image of the MASH catalogue.

PN G239.6-12.0: round with internal structure and intense patches. More images can be found in Fredrick \& West (1984).

PN G239.6+13.9: Balick (1989) classified this PN as elliptical (early-middle subtype). Additional images can be found in Schwarz et al. (1992).

PN G243.8-37.1: Boffin et al. (2012a) performed a morphological analysis of this PN.

PN G247.5-04.7: there is an HII region towards the SW, and it is unclear whether it is part of the PN or is the optical counterpart of the molecular cloud BRAN 63.
PN G249.3-05.4: ring-like PN with a very faint CSPN. It shows several irregularities in the brightness of the ring.

PN G249.8+07.1: this seems to be two overlapping PN. It is difficult to explain the homogeneous and extensive overdensity of material towards the NW.

PN G250.4-01.3: classical bipolar PN with an arc towards the NW. It is also visible in the [N II] images of Kerber et al. (2000).

PN G257.5+00.6: poorly studied planetary nebula with intriguing morphology. The PN displays a ring-like structure of low surface brightness towards the NE and a emission region towards the SSE that is difficult to explain. It probably is a bipolar PN. This object requires a more detailed study.

PN G258.0-15.7: Gonçalves et al. (2002) show an [N II] image, but it is not as deep as ours. In both cases the huge jet-like features are clearly visible. Additional images can be found in Corradi et al. (1996, 1999).

PN G259.1+00.9: it is difficult to perform a morphological classification of this Type I PN. A complete analysis of this object was made by Jones et al. (2014). Our [N II] image does not show any differences with the $[\mathrm{N}$ II] $+\mathrm{H} \alpha$ published in that article. A H $\alpha$ image is presented by Górny et al. (1999).

PN G260.7-03.3: the $\mathrm{H} \alpha$ image of MASH catalogue displays a more extended HII region than our image. According to its morphology, it would not be a PN. It could be a filament of the supernova remnant (SNR) Puppis A (Goudis \& Meaburn 1978).

PN G261.6+03.0: Corradi \& Schwarz (1995) classify this Type I PN as a possible/probable bipolar. Our [N II] image shows a well-defined bipolar morphology with a pronounced waist, thus we confirm this classification. It is not evident from our image if the two saturated regions define the torus of the waist. If this were the case, the waist would not be perpendicular to the bipolar lobes. To answer this question, it is necessary to perform a detailed spectroscopic study.

PN G261.9+08.5: this Type I PN has been classified by Peimbert \& Torres-Peimbert (1983) as a filamentary bipolar. More images can be found in Vázquez (2012), Bohigas (2003) and Dufour (1984).

PN G262.6-04.6: it presents a roughly round shape with two intense oval patches. Additional images can be found in Longmore \& Tritton (1980) and Schwarz et al. (1992).

PN G263.3-08.8: this object does not have published narrow-band images. Our image shows a round morphology with a dark band crossing the core, with a PA of $135^{\circ}$. Additional images can be found in Fredrick \& West (1984).

PN G264.1-08.1: well-defined elliptical nebula with a high surface brightness and an evident central star.

PN G265.1-04.2: ring-like PN with some knots on the edge. It is unclear if the off-centre bright star is indeed its ionizing source. Additional images can be found in Fredrick \& West (1984).

PN G265.4+04.2: round PN with two diametrically opposed condensations. Probable bipolar PN.

PN G268.6+05.0: object with no obvious symmetry. It displays an oval ring-like internal structure with opposite faint ansae. It might be a bipolar PN.

PN G268.9-00.4: this fascinating object has the appearance of a steering wheel. There is a nebulosity in the NW that is not visible in the MASH H $\alpha$ image. It is unclear if it belongs to the nebula. The central bright star could be the ionizing source. This object deserves a more detailed study.

PN G274.6+03.5: round PN with a bright bar along its diameter. Additional images can be found in Górny et al. (1999). 
PN G276.1-03.3: object that probably has a double envelope.

PN G277.1-03.8: López et al. (1989) have classified this PN as an evolved bipolar, but Louise et al. (1987) have classed it as peculiar. It has an overall bow-shock morphology, which was also noted by Rauch et al. (1999). Perhaps the gaseous envelope has interacted with the ISM. A detailed analysis of this object was presented by Lopez et al. (1991). More narrow-band images can be found in Drew et al. (2014), Perinotto \& Corradi (1998), Corradi \& Schwarz (1995), and Lopez et al. (1991).

PN G277.7-03.5: a round morphology with a complex ionization structure was revealed in our image. This object deserves a more detailed study.

PN G278.5-04.5: this object has been very poorly studied. Our image shows an elliptical envelope with an internal structure of point-symmetric type. In addition, we observe two pairs of beams in the E-W direction, similar to those of the Egg nebulae.

PN G279.1-03.1: roughly elliptical with two knots diametrically opposed.

PN G279.6-03.1: bipolar (Corradi \& Schwarz 1995), intense knots are superimposed at each end of the minor axis. In addition, it has a pair of arcs that make it an S-shaped nebula (Lee et al. 2007b). A detailed study of this object has been made by Corradi \& Schwarz (1993b). More images can be found in Lee et al. (2007a), Perinotto \& Corradi (1998) and Schwarz et al. (1992).

PN G280.0+02.9: round or point-like nebula. We do not detect any outer structure.

PN G285.5-03.3: strange morphology for a PN. Perhaps it is indeed the galaxy LEDA 2792457 and/or LEDA 2792455.

PN G286.3-00.7: Smith et al. (2007) showed a [N II]+H $\alpha$ narrow-band image of this PN. It is a clear ring PN.

PN G286.3+02.8: ring-like PN with a bright condensation to the SW.

PN G288.4-01.8: bipolar, it seems that its waist is a torus.

PN G288.4+00.3: it probably has two symmetrical jets at PA $100^{\circ}$.

PN G288.9-00.8: possible WR star of population I (Smith et al. 1994). Additional images can be found in Stahl (1987) and Frew et al. (2014).

PN G290.1-00.4: clear bipolar morphology with an bright waist. Moreover, in our image two ansae perpendicular to the minor axis are evident, which are not clear in the image published by Hua et al. (1998).

PN G290.5+07.9: the central star is visible. Well-studied object (see Palmer et al. 1996; Lopez et al. 1993), deeper images can be found in Boffin et al. (2012b).

PN G292.6+01.2: very bright PN with a complex internal structure. Bipolar appearance with a faint external shell. More images can be found in Górny et al. (1999).

PN G293.6+01.2: possible bipolar, with a very brilliant waist.

PN G293.6+10.9: well-defined irregular disc with a central hole. A double-shell structure can be seen. Aditional images can be found in Rauch et al. (1999).

PN G294.6+04.7: very complex object that has been studied in detail. It shows jets and knots (Corradi et al. 1999), an extended halo (Corradi et al. 2003, 2004), and a bow-shock feature (Guerrero et al. 2013). Additional narrow-band images can be found in Schwarz et al. (1992) and Richer et al. (1991).

PN G294.9-00.6: round morphology, an exterior elliptical structure may be present $\left(\mathrm{PA}=0^{\circ}\right)$. The central region is probably obscured by dust.
PN G296.6-20.0: elliptical morphology that resembles the Owl nebula.

PN G298.2-01.7: Corradi \& Schwarz (1995) classified it as a probable or possible bipolar PN. Our image allows us to confirm this classification. More images can be found in Górny et al. (1999).

PN G299.0+03.5: roughly elliptical with a bright waist. The central star is evident.

PN G299.0+18.4: ring-like with a diffuse double-shell; the central star is visible. Additional narrow-band images can be found in Schwarz et al. (1992).

PN G302.6-00.9: object poorly studied even though it displays a curious morphology. The PN exhibits an elliptical ringlike main structure. It seems to have a pair of symmetric jets in $\mathrm{PA}=135^{\circ}$.

PN G304.2+05.9: a ring-like object with a strange arc towards the NW. This is a very poorly studied object, it requires a more detailed analysis.

PN G307.2-03.4: PN with a puzzling morphology and a very hot [WO1] central star. Phillips \& Reay (1983) reported five sets of ansae, but Reay et al. (1984) described this situation as a ring of condensations. Zuckerman \& Aller (1986) classified this PN as irregular (with condensations and filaments), but Corradi \& Schwarz (1995) classified it as a probable or possible bipolar PN and Pascoli (1990) as a late butterfly. Finally, Sabin et al. (2012) performed a detailed study of this object and classified it as a quadrupolar nebula with multiple sets of symmetrical condensations. Moreover, a spherical halo is evident in the [O III] image presented by Hua et al. (1998). In our image we can distinguish two mass-loss events. The first is an irregular structure of intense knots, and the other is an internal and discontinuous bar. More images can be found in Górny et al. (1999), Polcaro et al. (1997), Perinotto et al. (1994), and Greve \& van Genderen (1987).

PN G309.0-04.2: we agree with the morphological description of Stanghellini et al. (1993a), who classified it as an elliptical with internal structure. Moreno et al. (1991) reported a nebulosity to the NW that we do not see. More images can be found in Kaler et al. (1989) and Schwarz et al. (1992).

PN G309.2+01.3: this PN is interacting with the ISM (Ali et al. 2012).

PN G310.7-02.9: narrow-band images are provided in Górny et al. (1999) and Moreno et al. (1991).

PN G310.8-05.9: round PN. More narrow-band images are shown in Longmore \& Tritton (1980).

PN G311.7-00.9: bipolar, the waist looks like a torus. The central star is evident.

PN G315.0-00.3: the $\mathrm{H} \alpha$ image from Górny et al. (1999) shows a condensation towards the NW that is not visible in our image. Other images are presented by Moreno et al. (1991), Meaburn \& Walsh (1989), and Moreno et al. (1987).

PN G317.1-05.7: the MASH H $\alpha$ image shows an external envelope that is not visible in ours. On the other hand, the $\mathrm{H} \alpha$ image (Moreno et al. 1991) does not reveal the internal structure that appears in our [N II] image. In the [O III] image (Górny et al. 1999) none of these structures are visible. Other narrow-band images can be found in Moreno et al. (1987).

PN G317.8+03.3: poorly studied PN that presents a ring-like inner structure with several irregularities in the brightness. A diffuse nebulosity is superimposed at each end of the minor axis.

PN G318.3-02.0: all authors agree with the classsification of this PN as bipolar. More images can be found in Górny et al. (1999), Corradi \& Schwarz (1995), Moreno et al. (1991), Louise et al. (1987), and Moreno et al. (1987). 
PN G318.3-02.5: well-defined elliptical nebula with two intense patches. More images can be found in Górny et al. (1999), Moreno et al. (1991, and 1987).

PN G318.4-03.0: ring-like PN.

PN G319.6+15.7: Type I PN with a [WR] core. This $\mathrm{PN}$, according to its appearance, is a typical bipolar, but Zuckerman \& Aller (1986) described this object as a disc of approximately uniform brightness, elliptical shape, and ansae. Hua et al. (1998) reported some internal structures. Several images of this PN have been taken in multiple works, for example O’Dell et al. (2002), Zhang \& Kwok (1998), Corradi \& Schwarz (1995), and Schwarz et al. (1992).

PN G321.8+01.9: ring-like type with a clear ansae to the W. Moreover, two symmetric condensations towards $\mathrm{N}$ and $\mathrm{S}$ are visible. We classify this object as a PE according to the classification scheme of Gorny et al. (1997). On the other hand, this PN has been classified as elliptical by Phillips (2003). Additional narrow-band images can be found in Górny et al. (1999).

PN G322.4-02.6: in the $[\mathrm{NII}]+\mathrm{H} \alpha$ image published by Marston et al. (1998), the knots at the edge of the object towards the NW are not visible, but they are clear in our [N II] image and may be LIS. H $\alpha$ and [N II] images shown by Schwarz et al. (1992) are overexposed. They do not show the complex internal structure that is evident in our [N II] image.

PN G327.8-07.2: the $\mathrm{H} \alpha$ image shows an elliptical ring of a faint nebulosity (Moreno et al. 1991). Our [N II] image is similar to the [O III] image of Moreno et al. (1991), showing a disc-like $\mathrm{PN}$. In addition, a faint nebulosity to the $\mathrm{N}$ and $\mathrm{S}$ of the inner region is visible in both images.

PN G328.9-02.4: round PN, the diffuse nebulosity to the $\mathrm{S}$ that we see in our image is not visible in the [O III] images shown by Górny et al. (1999). It may be an ultracompact HII region (Walsh et al. 1997). There is a young stellar object candidate near to this object, reinforcing the hypothesis of an HII region.

PN G329.3-02.8: the $\mathrm{H} \beta$ image (Górny et al. 1999) does not show the two $\operatorname{arcs}(\mathrm{N}$ and $\mathrm{S})$ visible in our [N II] image. This structure could be a LIS. The central star is not at the geometric centre, probably because of an interaction with the ISM.

PN G331.5-03.9: elliptical shape with internal structures. Additional images can be found in Górny et al. (1999).

PN G332.0-03.3: round shape with several irregularities in the brightness. Additional images can be found in Górny et al. (1999).
PN G334.3-09.3: Gorny et al. (1997) classified this object as a bipolar PN, subclass BE, and we agree with this classification. On the other hand, Stanghellini et al. (1993a) classified it as elliptical with outer structure. The $\mathrm{H} \alpha$ image published by Schwarz et al. (1992) shows a round halo.

PN G340.8+10.8: diffuse nebulosity, elliptical shape. Two symmetric arcs are easily discernible superimposed at the edge of the nebula $\left(\mathrm{PA}=70^{\circ}\right)$. This feature is not evident in the $\mathrm{H} \alpha$ image published by Górny et al. (1999).

PN G341.8+05.4: our image is different from the $\mathrm{H} \alpha$ image published by Górny et al. (1999), perhaps because of the seeing effects. It is difficult to perform a morphological classification, although it displays an elliptical shape (Gorny et al. 1997). We can distinguish in our image an internal structure. More images can be found in Liu et al. (2000) and Pottasch et al. (1986).

PN G342.7+00.7: it is probably a bipolar PN.

PN G342.9-02.0: the $\mathrm{H} \alpha$ image (Górny et al. 1999) displays a double-shell nebulosity. We can see in our [N II] image that there is an arc towards the $S$, still far away from the nebula.

PN G342.9-04.9: this object shows a morphology similar to PM 1-242 (Miranda et al. 2009). Additional images can be found in Górny et al. (1999).

PN G345.4+00.1: ring-like internal structure, with an external faint shell, intense condensations are superimposed towards the SE, and we can distinguish a knot towards the NE in our image. Stanghellini et al. (1993a) classified it as elliptical with an internal structure, Gorny et al. (1997) classified it as a probable elliptical. Additional images can be found in Schwarz et al. (1992).

PN G346.9+12.4: taking into account the peculiar morphology of this bright nebula, it is surprising that it has been very poorly studied. It consists of a bright ring-like internal structure with intense knots that are superimposed at each end of the minor axis. In addition, this object has an outer shell structure that is not perpendicular to the former. Hua et al. (1998) suggested that it is a bipolar PN, but we are unable to confirm this assertion.

PN G352.9+11.4: round PN with a [WCL] core. The morphology of this objects together with its brilliant CSPN make it similar to a nebula around a WC star of population I. Additional images can be found in Schwarz et al. (1992).

PN G353.7-12.8: this object could be the galaxy IRAS 18232-4031 according to its spiral morphology. 


\section{Appendix B: Additional figures}
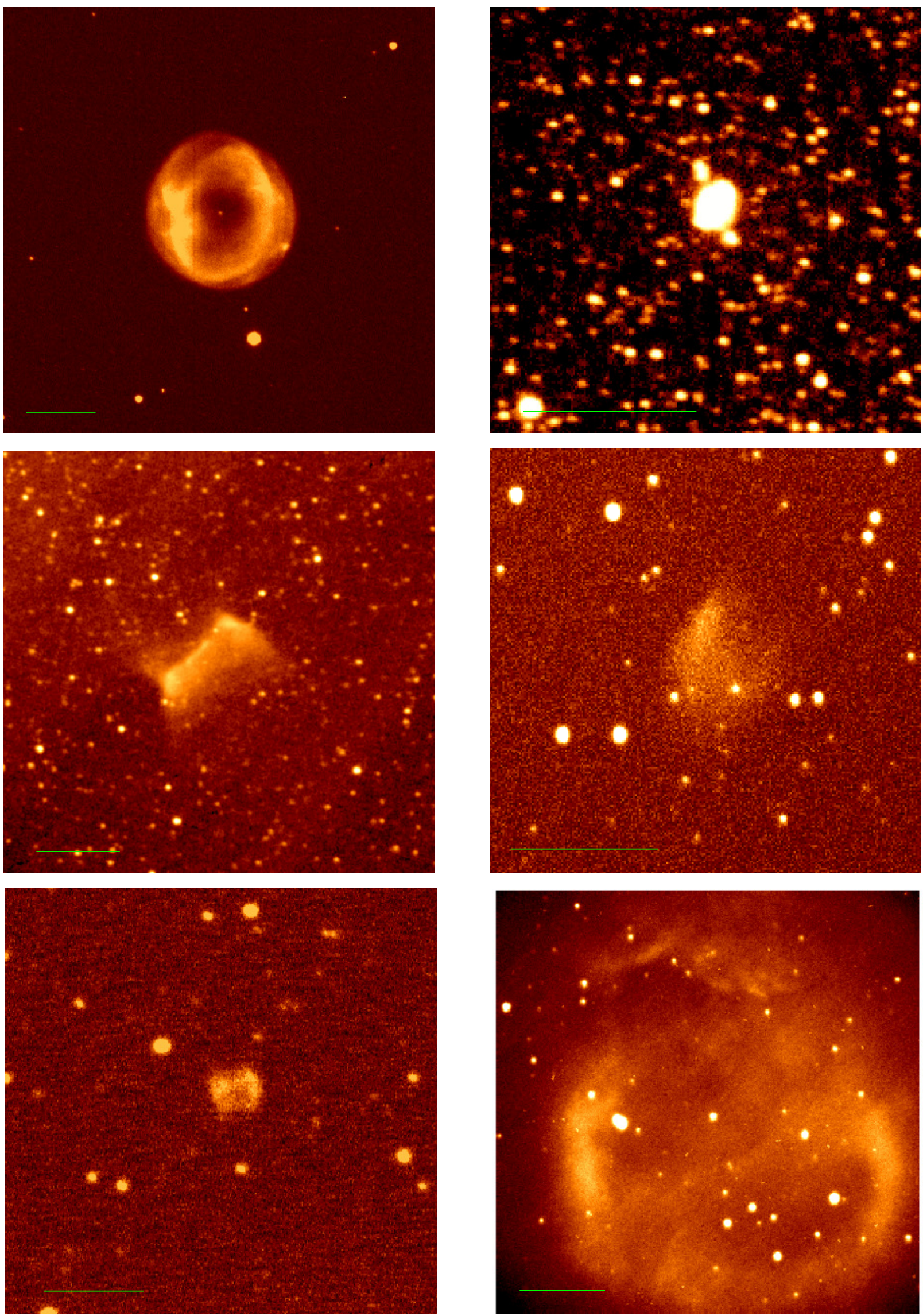

Fig. B.1. [N II] images of planetary nebulae. North is up and east to the left. The scale is indicated by a bar representing 60 arcsec. From left to right, top IC 5148-50 and Hb 4 (L), middle SaWe 3 and HDW 12, bottom DeHt 3 and A 66. 
W. A. Weidmann et al.: Atlas of monochromatic images of planetary nebulae $(R N)$
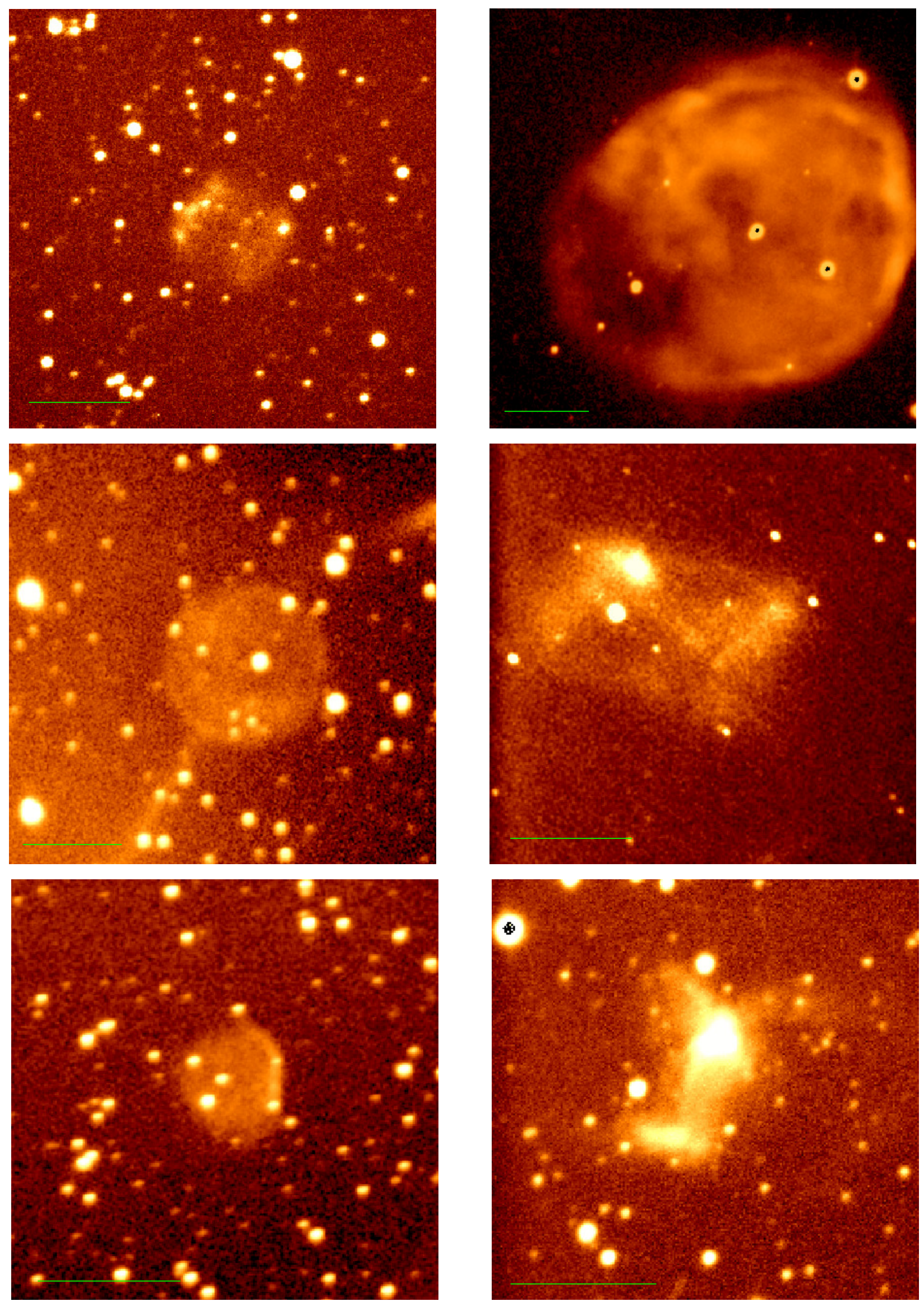

Fig. B.2. Same as Fig. B.1, top A 67 and NGC 246 (L), middle PHR0615-0025 (L) and K 1-11, bottom PHR0702-0324 (L) and PHR0705-0924 (L). 

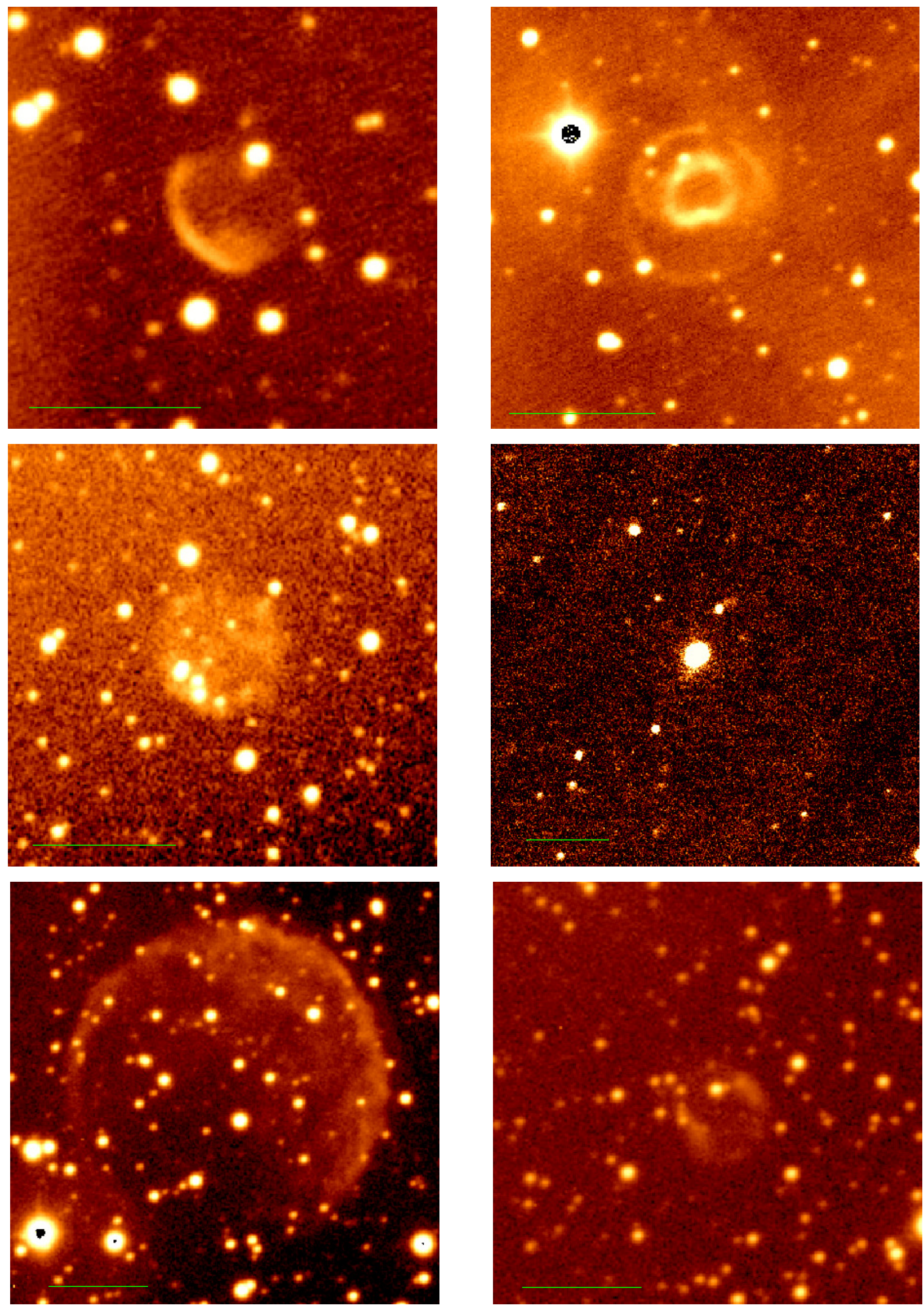

Fig. B.3. Same as Fig. B.1, top PHR0700-1143 (L) and PHR0716-1053, middle PHR0711-1238 (L) and M 1-6, bottom PHR0719-1222 (L) and PHR0727-1259 (L). 
W. A. Weidmann et al.: Atlas of monochromatic images of planetary nebulae $(R N)$
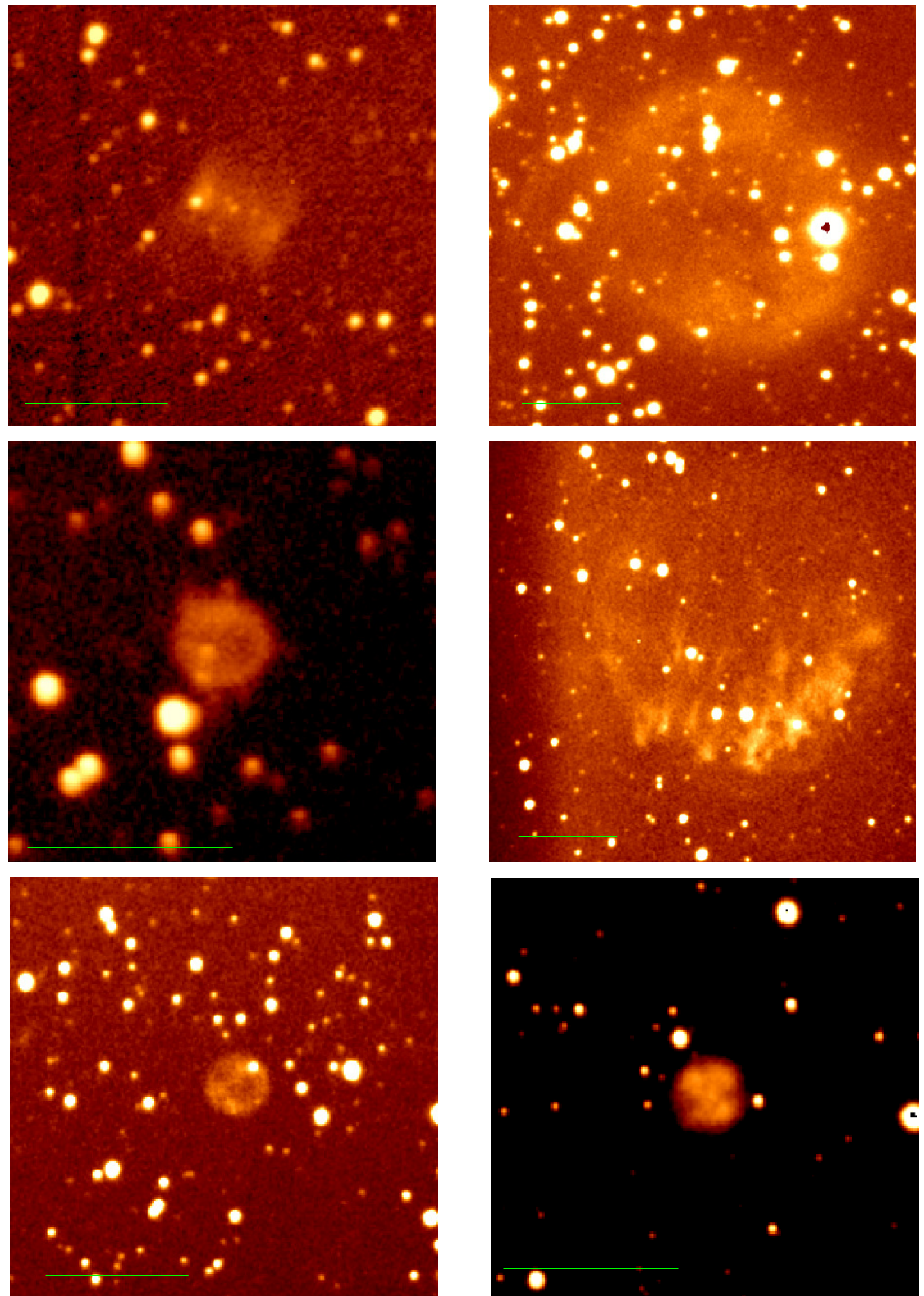

Fig. B.4. Same as Fig. B.1, top PHR0727-1707 (L) and PHR0724-1757, middle SaWe 1 and HaWe 9, bottom PHR0730-2151 and ESO $427-19(\mathrm{~L})$. 

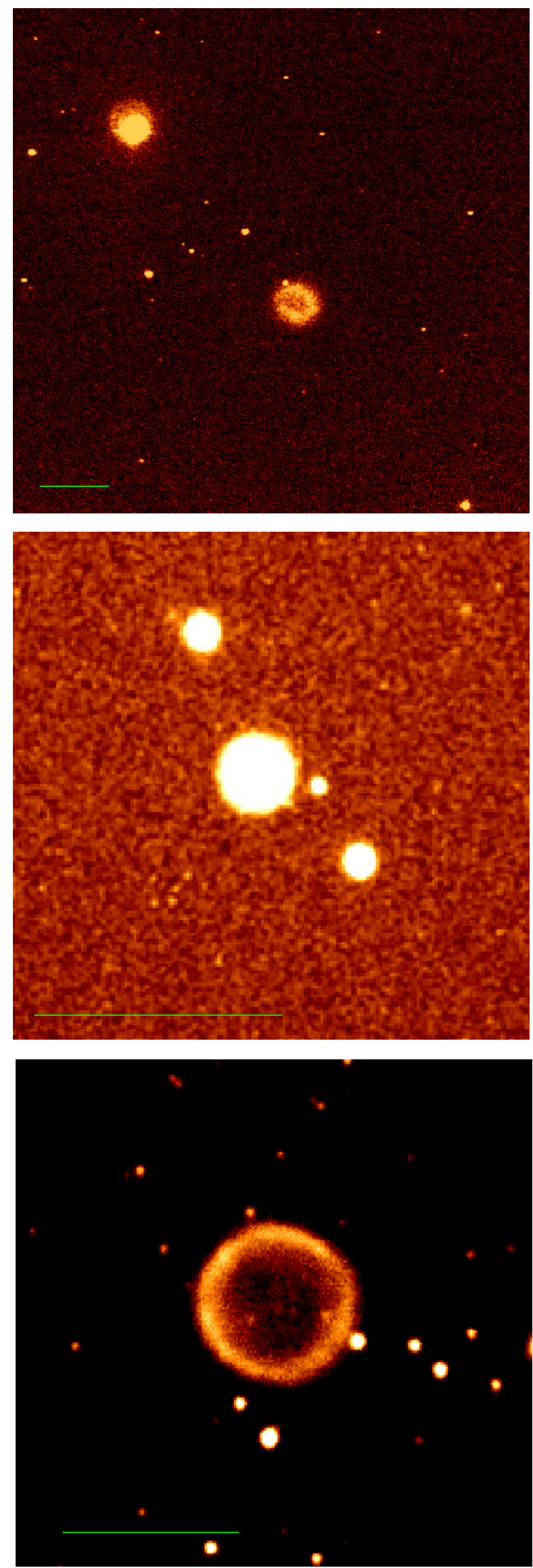

Fig. B.5. Same as Fig. B.1, top NGC 2610 and PHR0726-2858, middle PRTM 1 and PHR0742-3247, bottom A 23 and PHR0834-2819 (L).
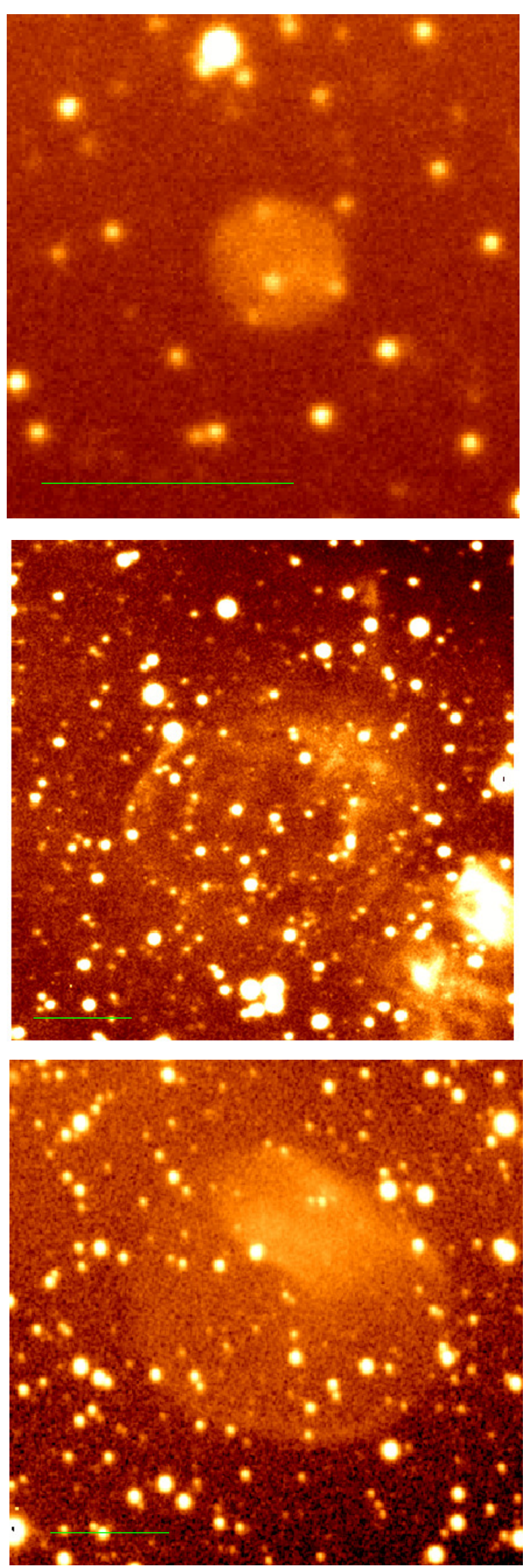
W. A. Weidmann et al.: Atlas of monochromatic images of planetary nebulae $(R N)$
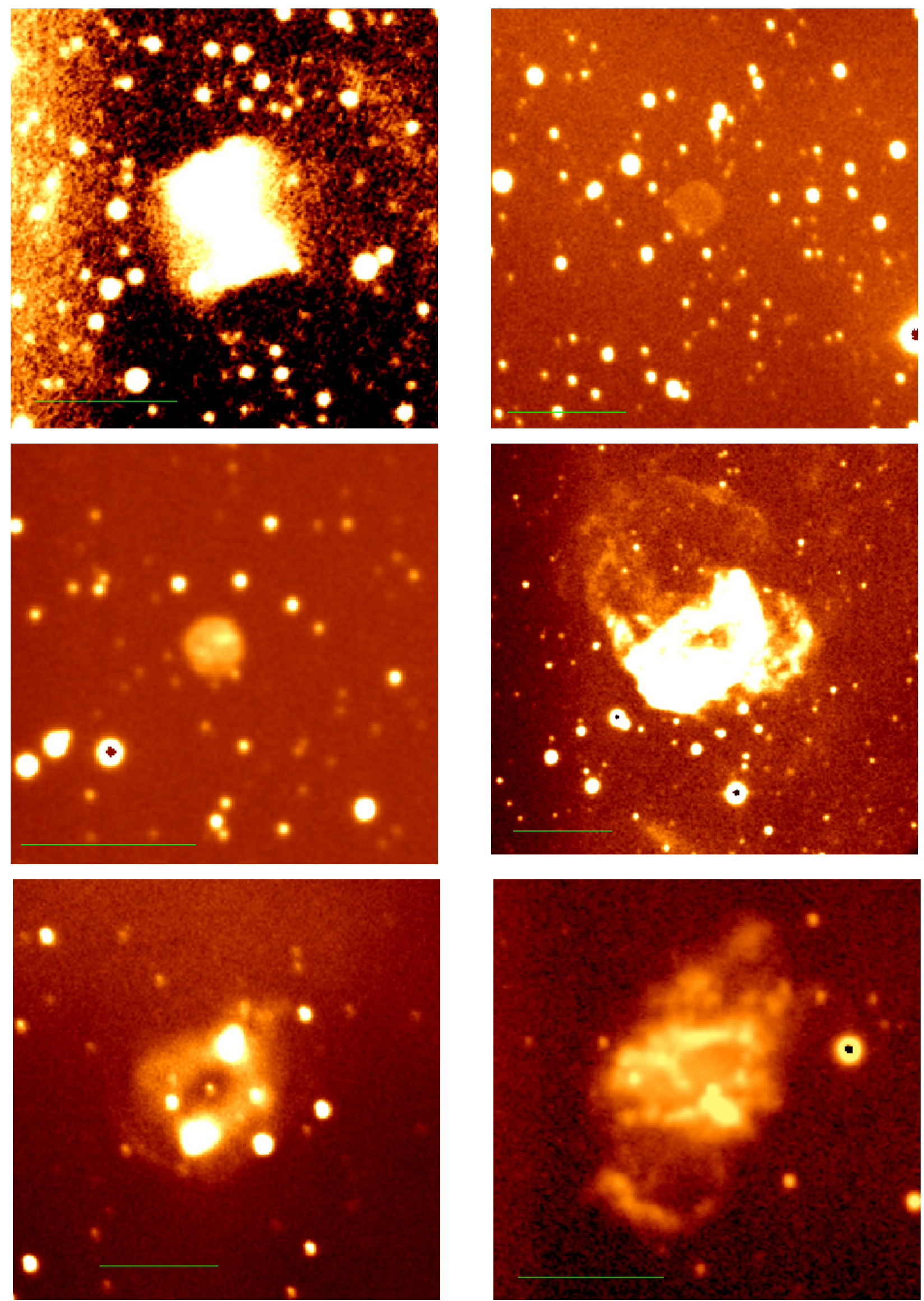

Fig. B.6. Same as Fig. B.1, top PHR0803-3331 (L) and PHR0736-3901, middle PHR0820-3516 and VBRC 1, bottom Wray 17-1 and He 2-11 (L). 
A\&A 592, A103 (2016)
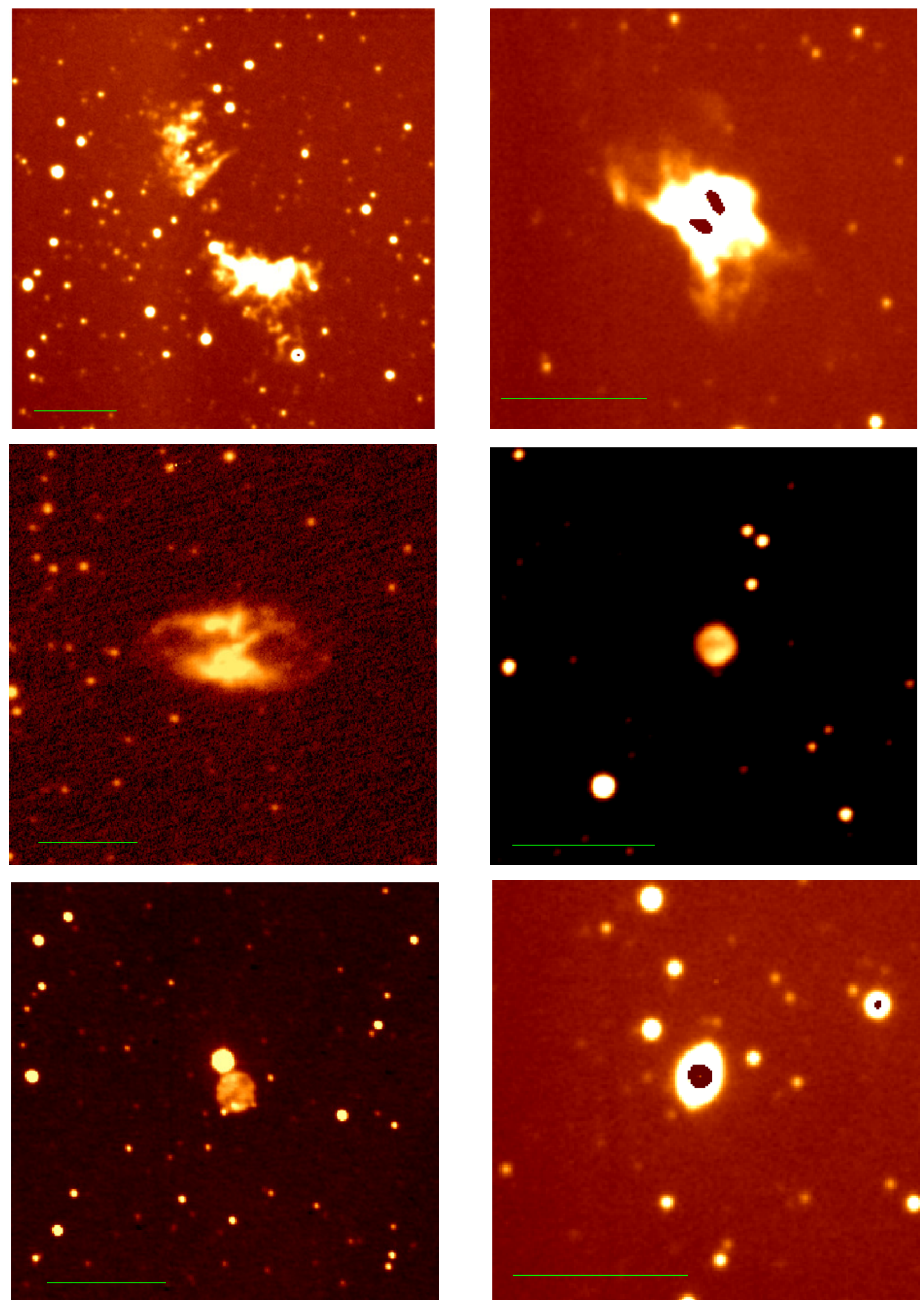

Fig. B.7. Same as Fig. B.1, top Wray 16-20 and He 2-15, middle NGC 2818 (L) and Wray 17-18 (L), bottom ESO 209-15 and He 2-7. 
W. A. Weidmann et al.: Atlas of monochromatic images of planetary nebulae $(R N)$
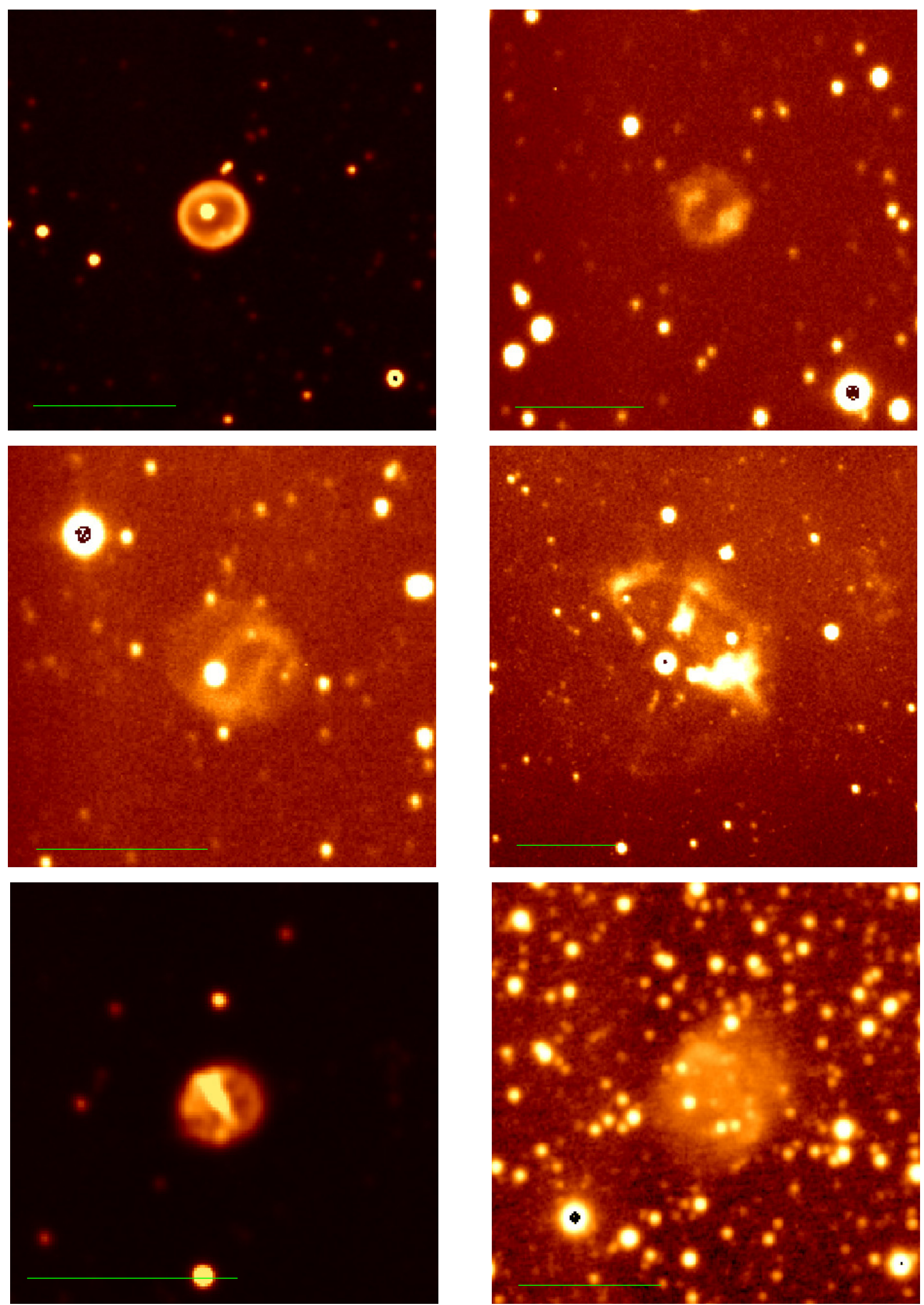

Fig. B.8. Same as Fig. B.1, top ESO 259-10 and PHR0911-4205, middle PHR0927-4347 and PHR0905-4753, bottom He 2-37 and PHR0924-5506. 
A\&A 592, A103 (2016)
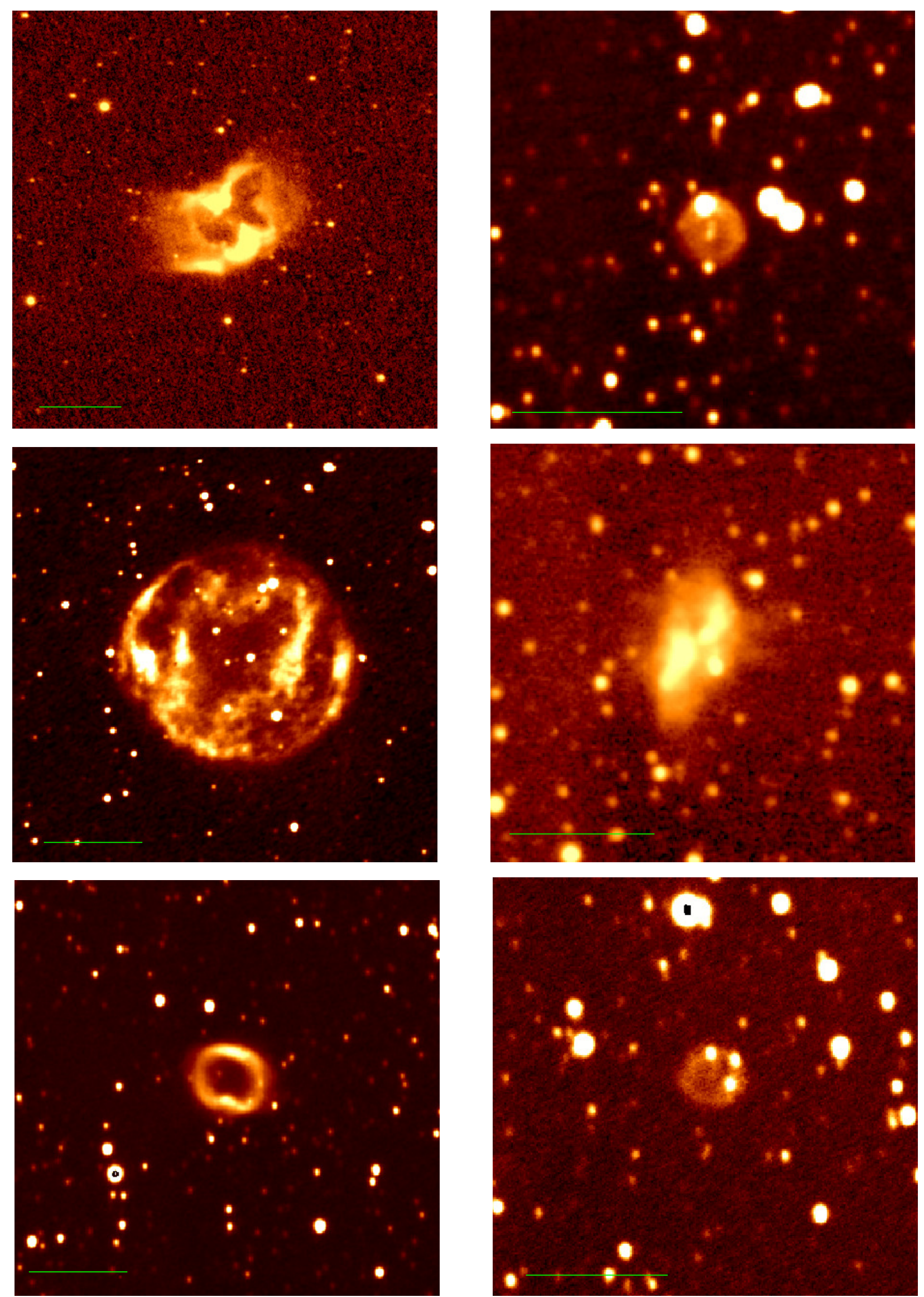

Fig. B.9. Same as Fig. B.1, top NGC 2899 (L) and PHR0958-5039, middle Wray 17-31 and He 2-32 (L), bottom PHR0940-5658 and PHR1010-5146. 
W. A. Weidmann et al.: Atlas of monochromatic images of planetary nebulae $(R N)$
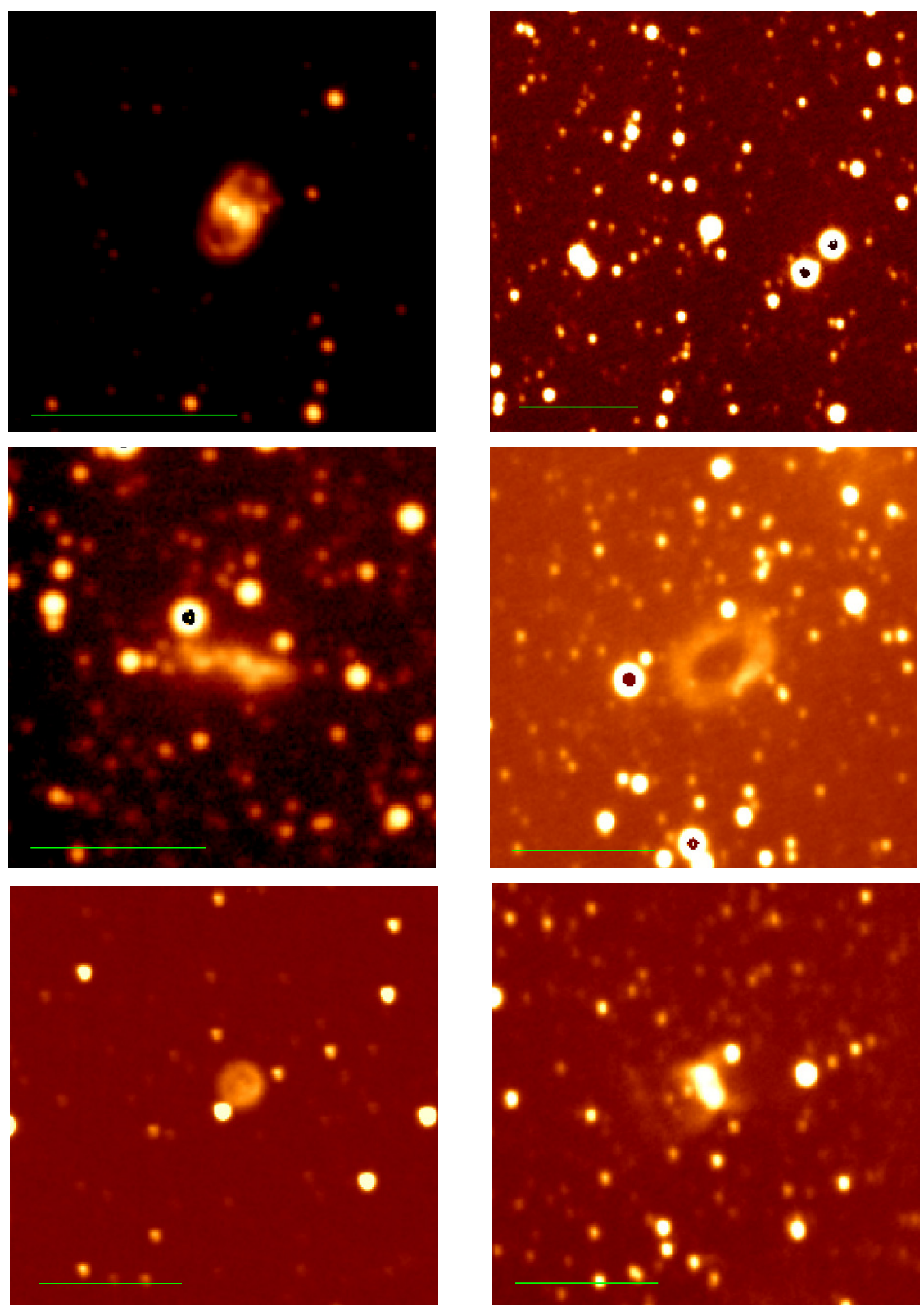

Fig. B.10. Same as Fig. B.1, top He 2-36 (L) and Ste 2-1, middle PHR1019-6059 (L) and PHR1036-5909, bottom He 2-55 and PHR1046-6109. 
A\&A 592, A103 (2016)
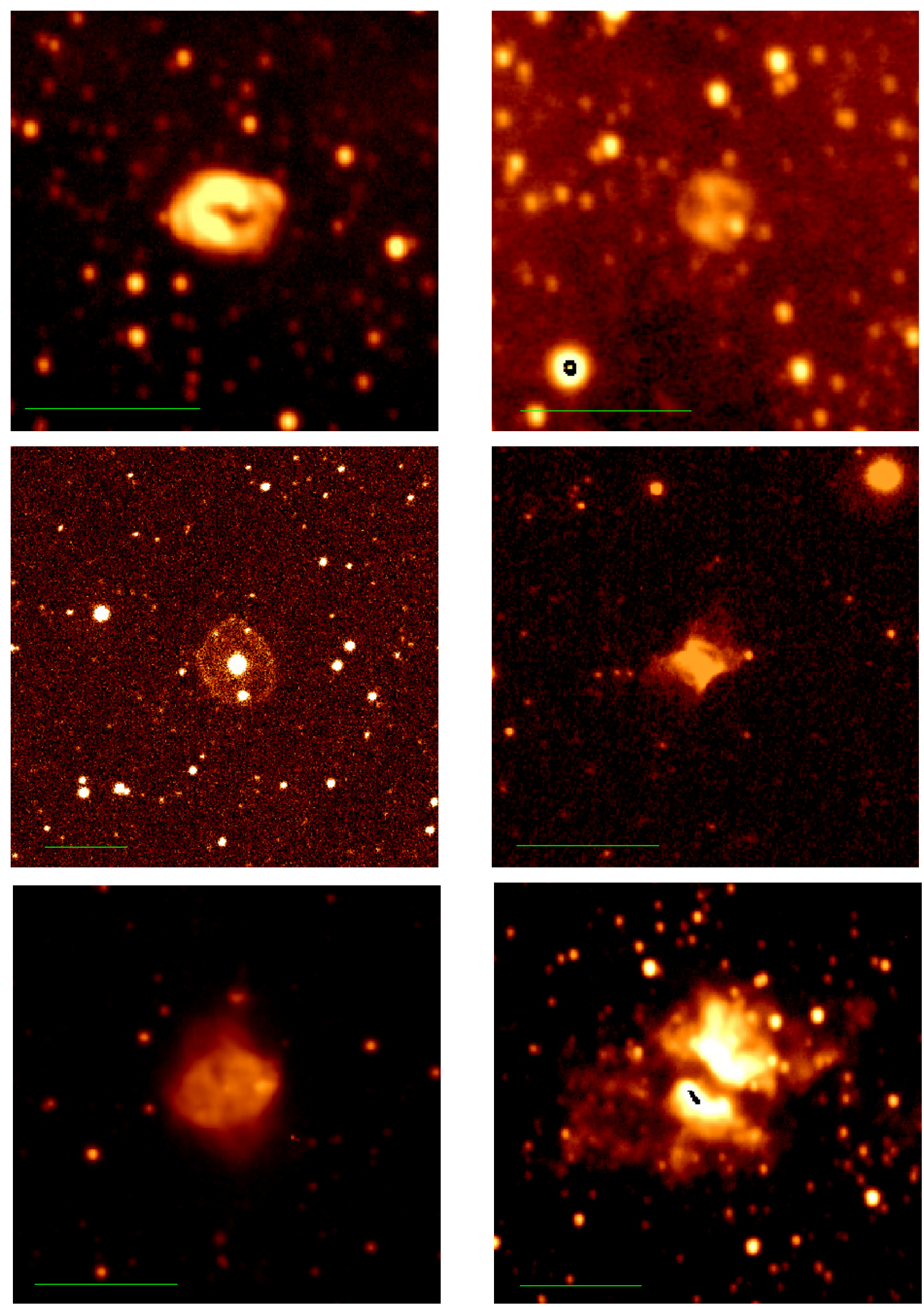

Fig. B.11. Same as Fig. B.1, top Hf 38 (L) and PHR1058-5853 (L), middle Hf 39 and Hf 48 (L), bottom Fg 1 (L) and NGC 3699 (L). 
W. A. Weidmann et al.: Atlas of monochromatic images of planetary nebulae $(R N)$
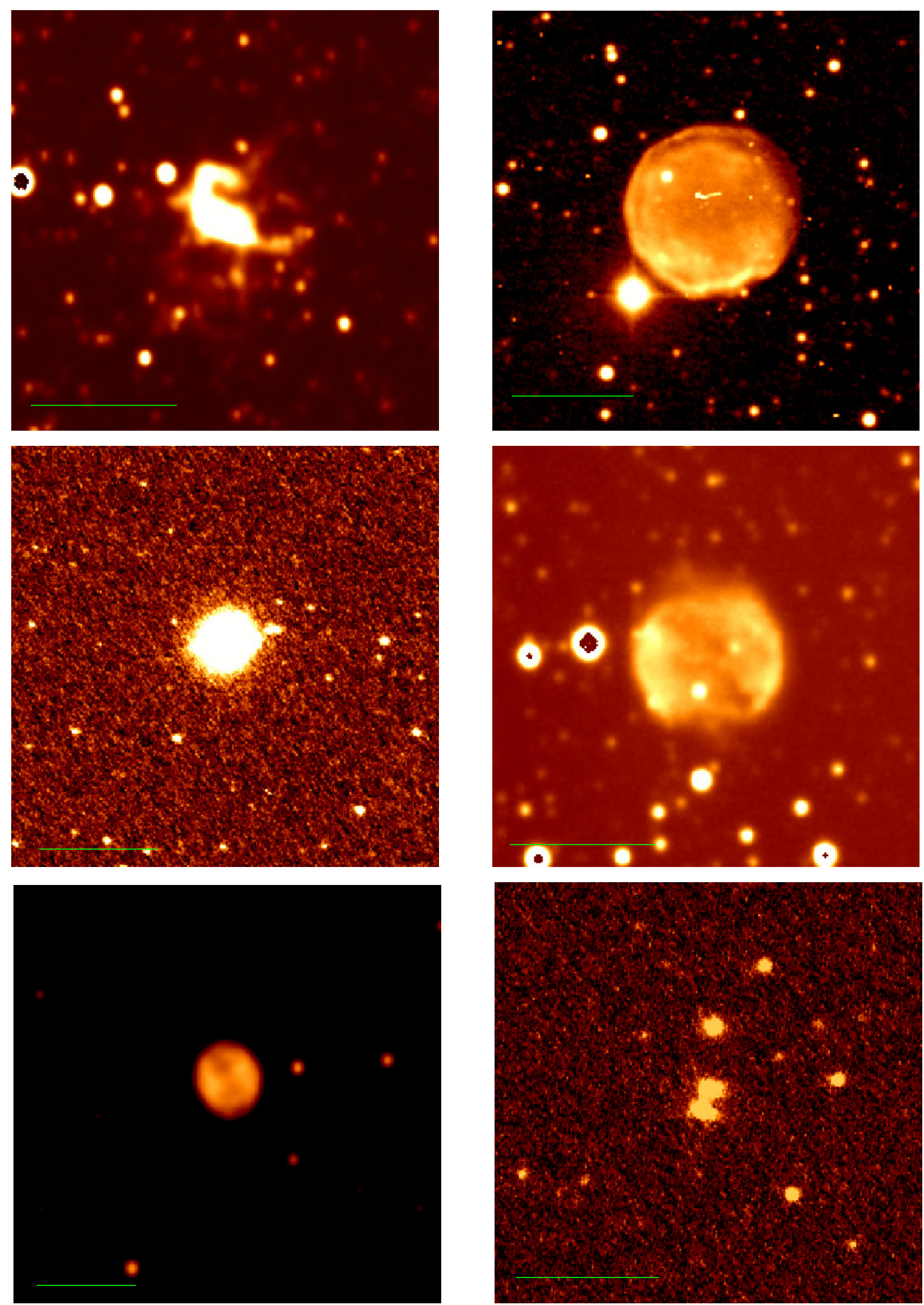

Fig. B.12. Same as Fig. B.1, top He 2-70 and BIDz 1, middle NGC 3918 and He 2-72, bottom NGC 3195 (L) and He 2-76. 

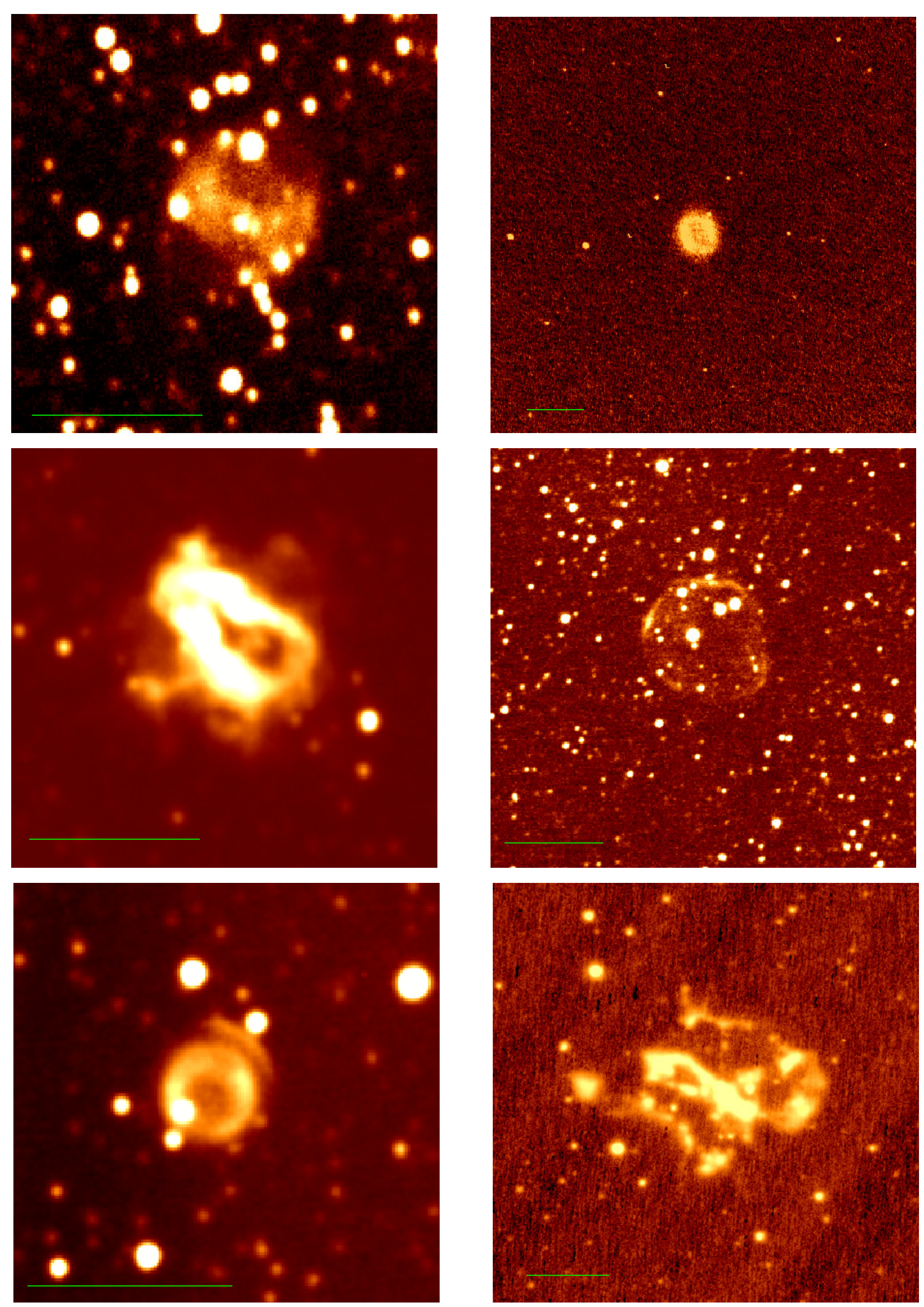

Fig. B.13. Same as Fig. B.1, top PHR1221-5907 and K 1-23, middle Wray 16-121, and PHR1250-6346, bottom Wray 16-122 and NGC 5189 (L). 
W. A. Weidmann et al.: Atlas of monochromatic images of planetary nebulae $(R N)$
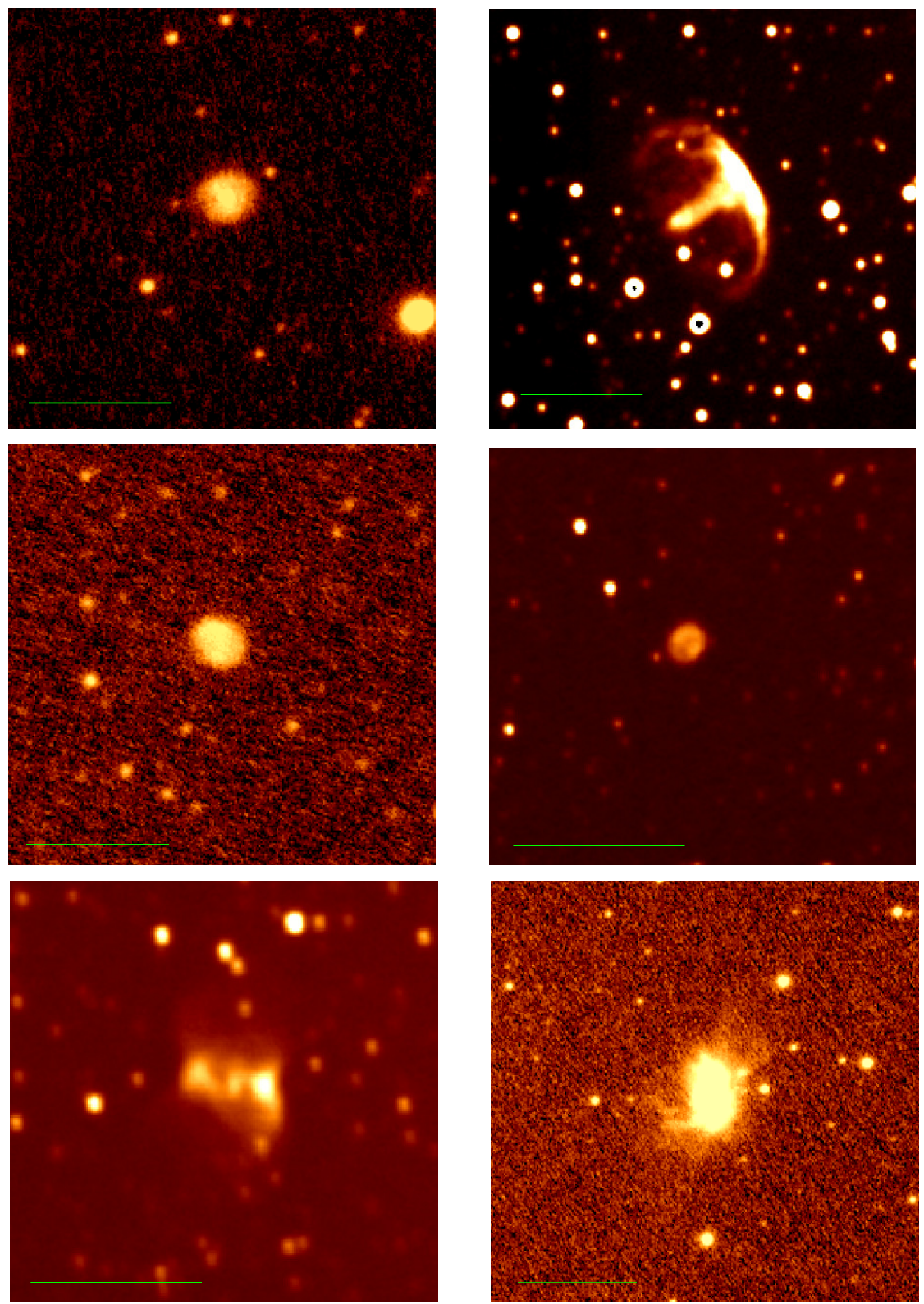

Fig. B.14. Same as Fig. B.1, top He 2-99 (L) and VBRC 5, middle He 2-103 (L) and LoTr 7, bottom PHR1408-6229, and He 2-111 (L). 
A\&A 592, A103 (2016)
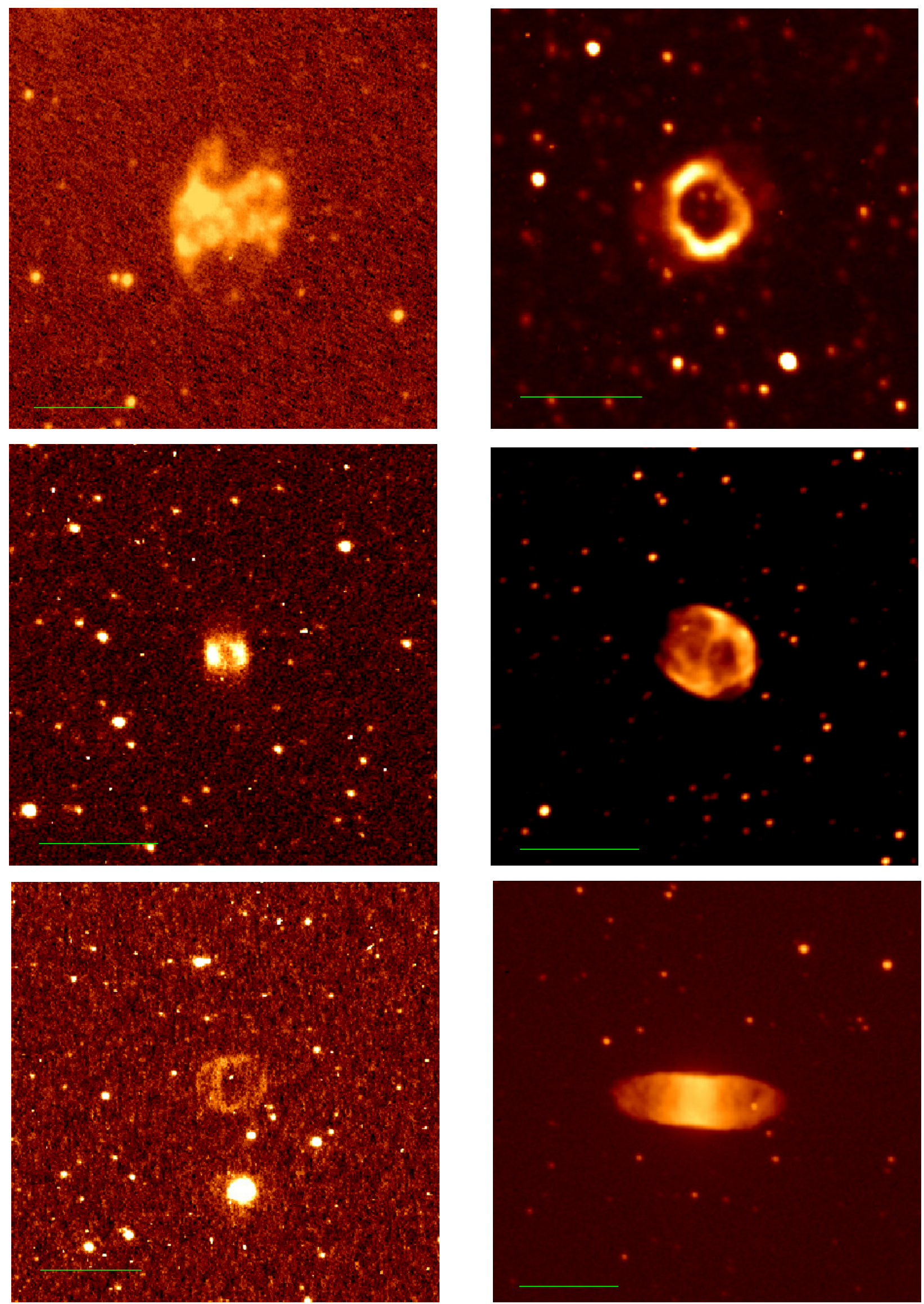

Fig. B.15. Same as Fig. B.1, top He 2-119 (L) and VBRC 6, middle He 2-114 and He 2-116 (L), bottom ESO $135-04$ and IC 4406 (L). 

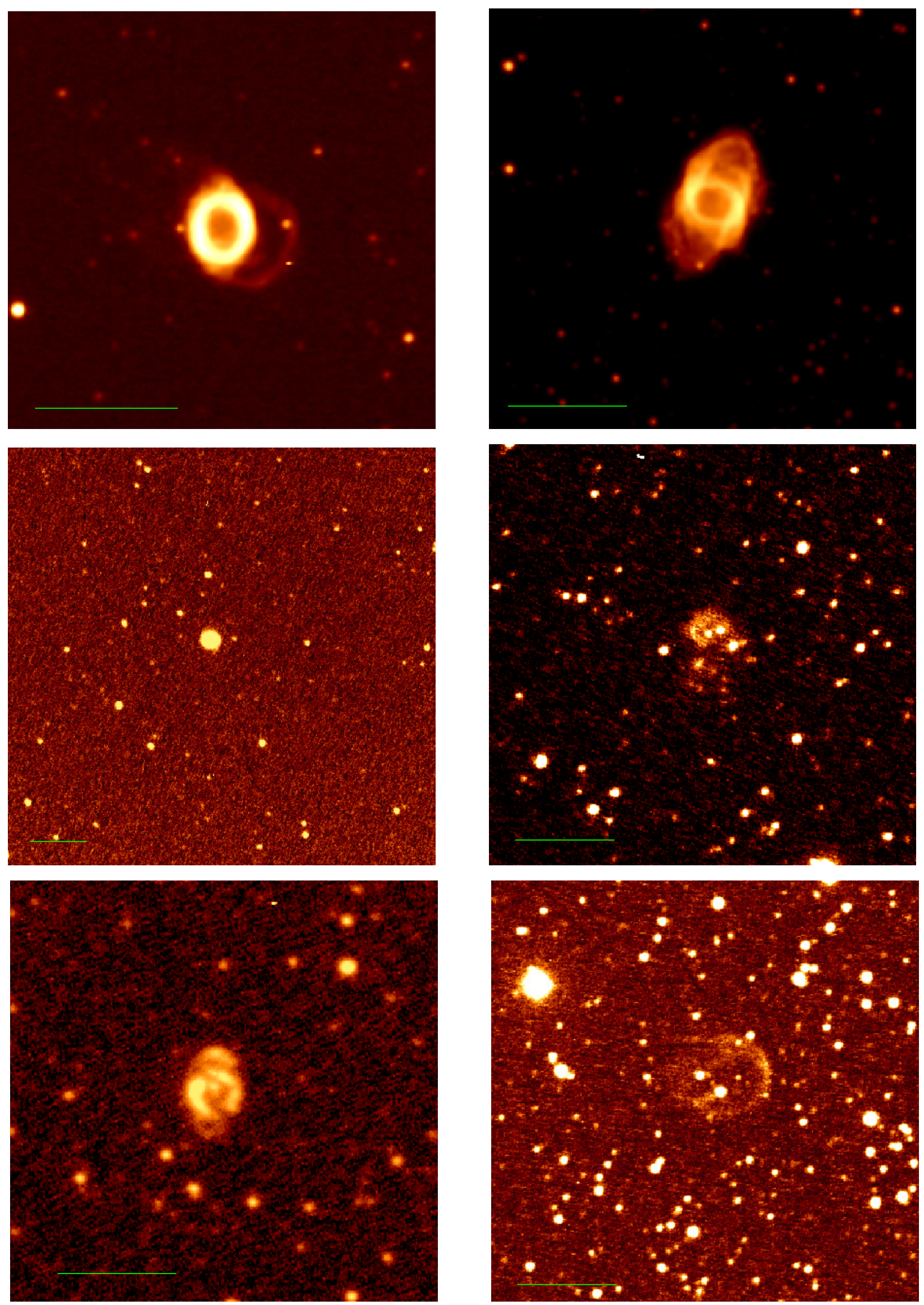

Fig. B.16. Same as Fig. B.1, top He 2-120 (L) and Mz 1 (L), middle He 2-163 and He 2-146, bottom Mz 2 (L) and PHR1557-5128. 
A\&A 592, A103 (2016)
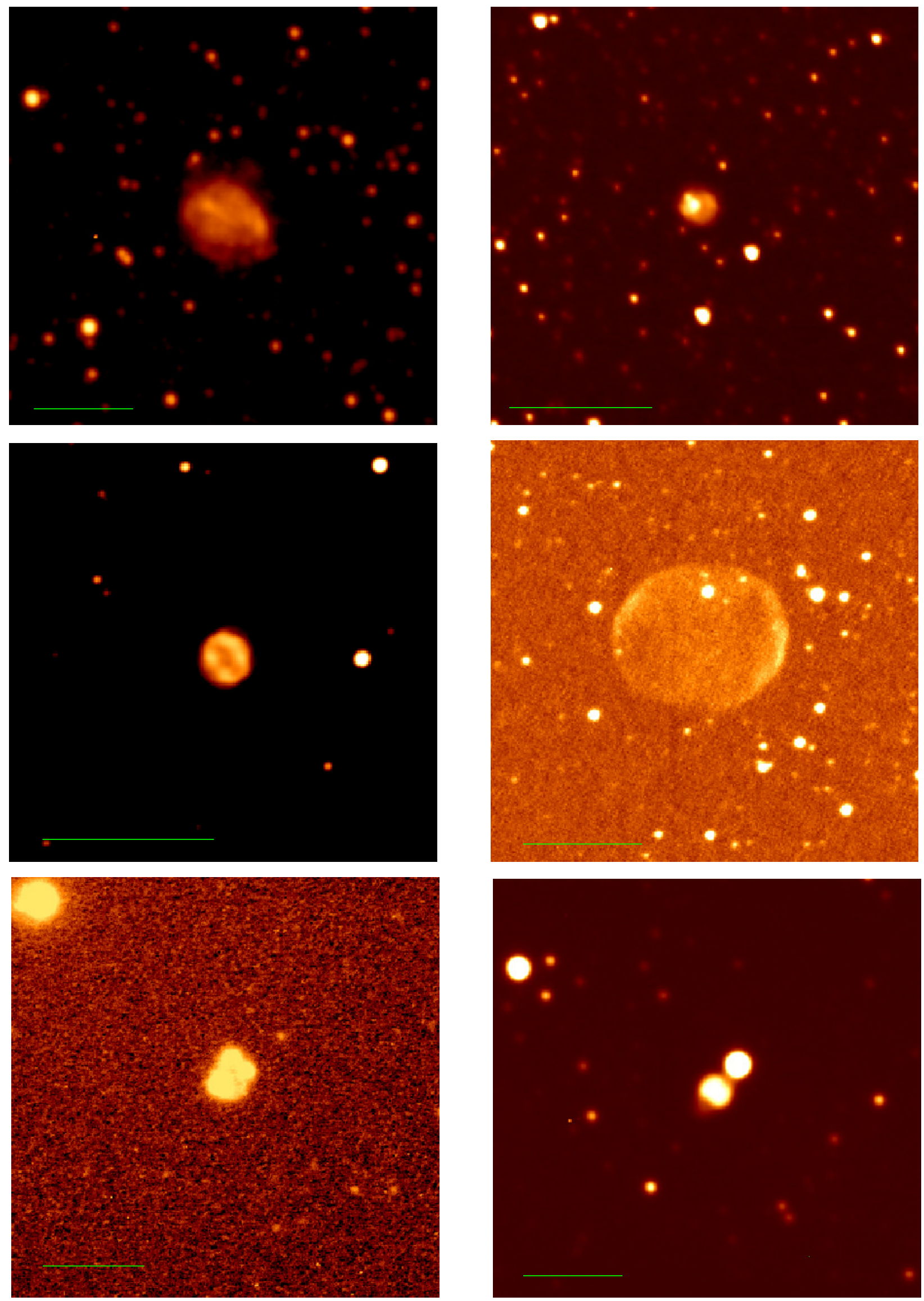

Fig. B.17. Same as Fig. B.1, top He 2-165 (L) and He 2-164, middle IC 4642 (L) and Lo 12, bottom NGC 6153 and H 1-3. 
W. A. Weidmann et al.: Atlas of monochromatic images of planetary nebulae $(R N)$
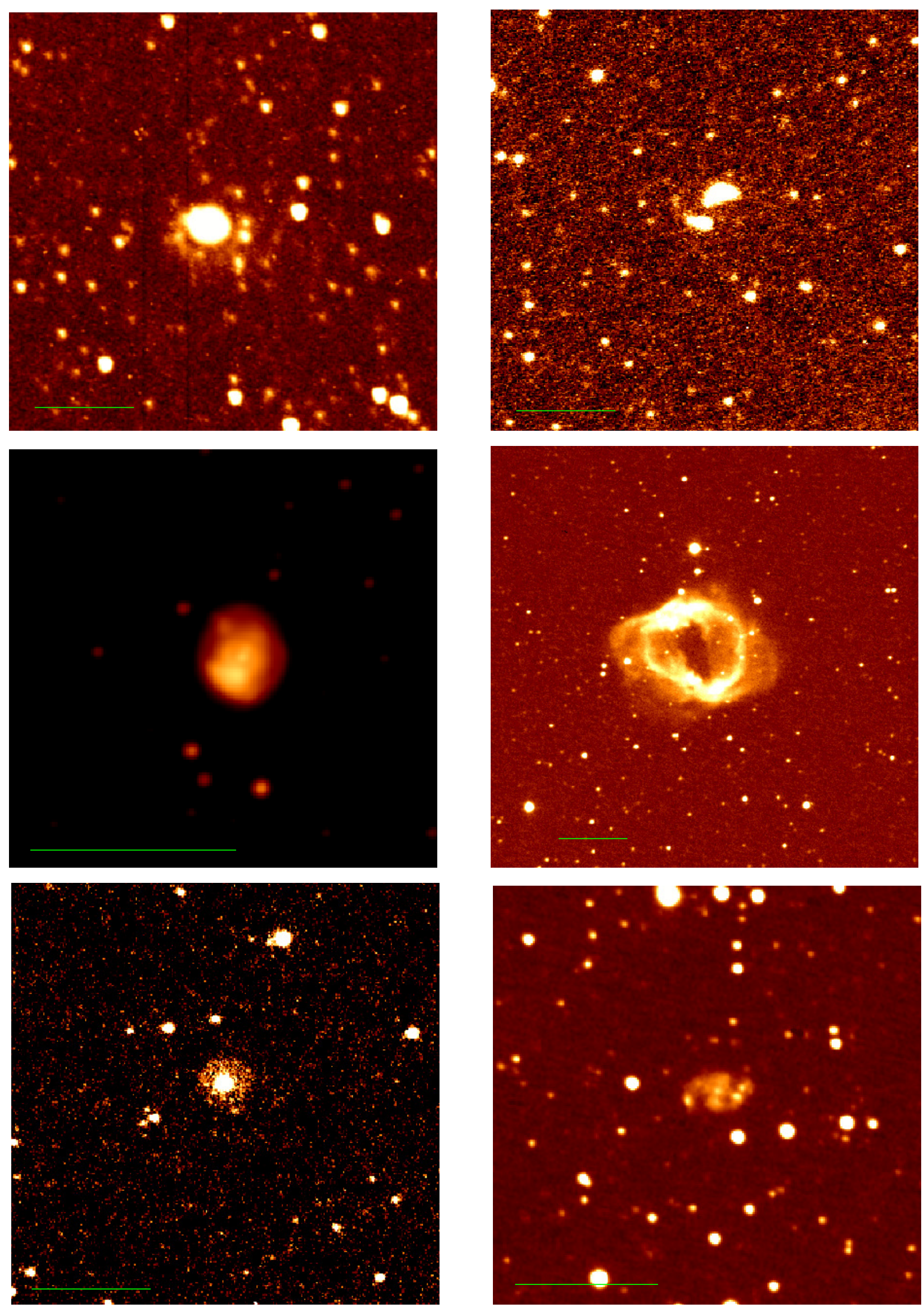

Fig. B.18. Same as Fig. B.1, top Pe 1-8 (L) and He 2-207, middle IC 4637 (L) and K 1-3, bottom K 2-16 (L) and Wray 16-411. 